\title{
An MCM-Enhanced Compressive Sensing for Weak Fault Feature Extraction of Rolling Element Bearings under Variable Speeds
}

\author{
Ya He, ${ }^{1}$ Kun Feng $\left(\mathbb{D},{ }^{2}\right.$ Minghui $\mathrm{Hu}\left(\mathbb{D},{ }^{1}\right.$ and Jinmiao Cui ${ }^{2}{ }^{2}$ \\ ${ }^{1}$ Beijing Key Laboratory of High-End Mechanical Equipment Health Monitoring and Self-Recovery, \\ Beijing University of Chemical Technology, Beijing 100029, China \\ ${ }^{2}$ Key Lab of Engine Health Monitoring-Control and Networking of Ministry of Education, \\ Beijing University of Chemical Technology, Beijing 100029, China \\ Correspondence should be addressed to Kun Feng; kunfengphd@163.com and Minghui Hu; humh2008@163.com
}

Received 19 September 2019; Revised 20 January 2020; Accepted 24 February 2020; Published 13 August 2020

Academic Editor: Rafał Burdzik

Copyright ( $) 2020$ Ya He et al. This is an open access article distributed under the Creative Commons Attribution License, which permits unrestricted use, distribution, and reproduction in any medium, provided the original work is properly cited.

\begin{abstract}
The compressive sensing (CS) theory provides a new slight to the big-data problem led by the Shannon sampling theorem in rolling element bearings condition monitoring, where the measurement matrix of CS tends to be designed by the random matrix (RM) to preserve the integrity of signal roughly. However, when the signal to be analyzed is infected with strong noise, not only does the signal become insufficiently sparse, but the randomness of the measurement matrix will bring down the sensing efficiency, resulting in the loss of fault feature. Thus, a sensing-enhanced CS scheme based on a series of modes after VMD decomposition is proposed under this paper. The core of this scheme is as follows: (1) the principal mode of VMD with better sparsity replaces the raw signal for compressive sensing; (2) all these modes contain the time-frequency characteristics of the raw signal; (3) a new measurement matrix called mode-circulant matrix (MCM) is defined by circulating the mode matrix, and when the amount of samples is shrunk, the sensing efficiency can be enhanced greatly. Besides, considering the fault signal of rolling bearings under variable speed, there is a need to use order tracking to overcome the nonstationarity of the signal before applying CS theory. The analysis results of simulation and experiment prove that the VMD- and MCM-based CS can successfully extract the weak fault feature of rolling bearings with operating speed changing.
\end{abstract}

\section{Introduction}

The condition monitoring of rolling element bearings is a crucial method used in a wide range of helping to prevent unpredictable failure. So far, vibration-based diagnosis is a valid and frequently used pattern of bearing health assessment $[1,2]$, and the increasing interest in vibration-based diagnosis has heightened the need for vibration analysis approaches suitable to extract the faulty feature of rolling bearings [3]. Of particular concern and significant are the time-domain analysis (time-synchronous averaging [4]; symptom parameters [5]), frequency domain analysis (resonance demodulation [6]; cepstrum analysis [7]), and time-frequency analysis (wavelet transform [8]; empirical mode decomposition $[9,10])$.

The cited literature demonstrated that the fault diagnosis of rolling bearings is explored increasingly. However, since almost all of the above analysis methods are based on signal sampling without loss, high sampling frequencies must be obliged to ensure the accurate acquisition of bearing fault feature under the Shannon sampling theorem [11]. It subsequently will generate enormous amounts of data exerting more pressure on the data storage, processing, and remote transmission in continuous condition monitoring of operating equipment. Therefore, it would be preferable for its working state to be identified quickly from less transferred data. This means when the equipment fails, it takes less time to diagnose and provide suggestions averting evolving into a fatal accident.

Compressive sensing (CS) has provided a fine insight for tackling this challenge. This method was primarily proposed by Candès et al. and Donoho [12, 13], whose main goal was to reconstruct the original signals from a small number of samples using sparse representation and a well-designed measurement matrix. The CS theory has been employed to 
image processing, nuclear magnetic resonance, remote sensing, radar, and so on. Built on CS, Chan et al. [14] implemented compression and reconstruction of an image under a small number of measurements, and the collection efficiency improved. Lustig et al. [15] studied that the time of data acquisition can be sharply reduced by MRI through CS technology. Xie [16] performed range compression by using the CS technique and a two-dimensional imaging scheme was established.

Owing to the excellent effect of CS theory in the application, it has attracted extensive attention from scholars in the field of fault diagnosis. Li et al. [17] attempted to compress and reconstruct monitoring signals of train rolling bearing; the results showed that the fault can be identified from the reconstructed signal. Zhang et al. [18] implemented a bearing fault detection method based on compressed sampling and matching track. As introduced by Chenet al. [19], sparse extraction of impulse by an adaptive dictionary algorithm based on the sparse model of CS can extract impulse components effectively. Tang et al. [20] developed a sparse representation classification strategy for condition evaluation of rotating machinery. Wang et al. [21] utilized a TQWT-based CS method to overcome the problem of inadequate sparsity and extract the faulty feature of rolling bearings. The aforementioned studies, based on CS theory, aimed at promoting the sparsity or reconstruction performance, but few kinds of literature are linked to measurement matrix optimization.

The measurement matrix is at the heart of signal compression and reconstruction of CS. Current literature on measurement matrix construction mainly focuses on the deterministic measurement matrix. Depending on the energy of each part of the signal, Guo et al. [22] designed a matching deterministic measurement matrix based on the principle of observation adaptive allocation. Based on the characteristics of the signal, Abtahi and Modarreshashemi [23] used a large number of comparable training signals to guide the construction of deterministic measurement matrixes. To optimize the measurement matrix, S.H.S. Masoumian and Tazehkand [24] proposed that the measurement matrix selected in the next iteration can be given in judging differential entropy reduction speed. In the literature about voice signal and image analysis, the results show that, compared with the random measurement matrix, the sensing efficiency of the deterministic measurement matrix has been greatly improved, which demonstrates the possibility of constructing a deterministic measurement matrix in the application of CS in fault diagnosis. When the CS is applied in fault diagnosis, random measurement matrices are mostly employed as dimension reduction to preserve the integrity of the signal with high probability [25]. However, owing to the randomness, useful and useless information will be acquired with equal probability resulting in low sensing efficiency. Besides, the fault diagnosis of rolling bearing in practical application still has the following challenges [2]: (1) the amplitude of vibration induced by initial faults in rolling bearings is very small; (2) the high natural frequency excited by faults of rolling bearings attenuates rapidly from the faulty bearing to vibration sensors; (3) due to complex transfer paths and complicated working environments, lots of noises are mixed in vibration signals collected in practice. All these factors combined will lead to the vibration caused by bearing failure which is extremely weak, in which the fault feature of bearing may be lost but the real redundant information may be retained with a random measurement matrix.

Therefore, a sensing enhanced method named the modecirculant matrix (MCM) is utilized in this study to increase sensing efficiency on vibration signals, which are advantageous to the weak fault feature of rolling bearings based on CS theory. Assisted by VMD [26, 27], the raw signal can be decomposed into a series of modes that contain the local characteristics of the original signal at different time scales. The MCM is constructed by the modes, and it can achieve higher sensing efficiency than the RM when CS is performed. Furthermore, it is well established that the quality of the CS application can be affected by the sparse representation of the signal. However, the inclusion of strong noise in the signal will seriously decrease the sparsity of the signal in the sparse dictionary. To promote the sparsity, the main mode is determined from a series of modes through VMD based on the principle of energy ratio [28], which can eliminate noise and highlight the faulty feature of bearing. Besides, with the help of order tracking, this method can be extended to extract fault feature of nonstationary vibration signals of rolling bearings running at variable speed. So a weak fault feature extraction scheme based on VMD and CS for rolling element bearings at variable speed is proposed.

The structure of this paper is arranged as follows. Section 2 presents the theoretical bases of VMD and CS. Section 3 describes the proposed scheme which adopts VMD and CS to construct the MCM and extract the weak fault feature of rolling bearings. Moreover, the detailed procedures of this method are discussed in this section. Section 4 establishes the model of defect signal collected at different locations of rolling bearing and simulates the fault signal of the inner race defect. Then, the effectiveness of the proposed scheme is demonstrated using simulated and experimental signals of rolling bearing faults in Sections 4 and 5, respectively. Section 6 presents the conclusions of the study.

\section{Basic Concept of the Proposed Feature Extraction Scheme}

2.1. Variational Mode Decomposition. VMD is an adaptive signal decomposition method. It can nonrecursively decompose the vibration signal into several quasiorthogonal modes. The optimal solution of the variational model can be used to obtain the frequency center and bandwidth of each mode and adaptively separate the mode components [26, 27].

To estimate the bandwidth of each mode, the constrained variational problem is constructed as shown in

$$
\begin{array}{ll}
\min _{\left\{u_{k}\right\},\left\{\omega_{k}\right\}} & \left\{\sum_{k}\left\|\partial_{t}\left[\left(\delta(t)+\frac{j}{\pi t}\right) \cdot u_{k}(t)\right] e^{-j \omega_{k} t}\right\|_{2}^{2}\right\} \\
\text { s.t. } \quad & \sum_{k} u_{k}=f,
\end{array}
$$


where $\left\{u_{k}\right\}:=\left\{u_{1}, \ldots, u_{k}\right\}$ and $\left\{\omega_{k}\right\}:=\left\{\omega_{1}, \ldots, \omega_{k}\right\}$ represent a series of modes and corresponding center frequencies, respectively.

The constrained problem can be simplified to the unconstrained variational problem by introducing quadratic penalty factor $\alpha$ and Lagrange multiplication operator $\lambda$. The augmented Lagrange multiplier is given as follows:

$$
\begin{aligned}
L\left(\left\{u_{k}\right\},\left\{\omega_{k}\right\}, \lambda\right):= & \alpha \sum_{k}\left\|\partial_{t}\left[\left(\delta(t)+\frac{j}{\pi t}\right) \cdot u_{k}(t)\right] e^{-j \omega_{k} t}\right\|_{2}^{2} \\
& +\left\|f(t)-\sum_{k} u_{k}(t)\right\|_{2}^{2} \\
& +\left\langle\lambda(t), f(t)-\sum_{k} u_{k}(t)\right\rangle .
\end{aligned}
$$

Equation (2) can be solved by the alternate direction method of multipliers. In other words, the optimal solution of the constrained variational problem can be found through updating $u_{k}^{n+1}, \omega_{k}^{n+1}, \lambda^{n+1}$ alternately.

The procedure of VMD can be described as follows:

(1) Initialize $\left\{\hat{u}_{k}^{1}\right\},\left\{\omega_{k}^{1}\right\}, \lambda^{1}, n \leftarrow 0$.

(2) Repeat $n=n+1$.

(3) $k=k+1$, until $k=K$. Update $u_{k}$ :

$$
\widehat{u}_{k}^{n+1}(\omega) \longleftarrow \frac{f(\omega)-\sum_{i<k} \widehat{u}_{i}^{n}(\omega)+\left(\lambda^{n}(\omega) / 2\right)}{1+2 \alpha\left(\omega-\omega_{k}^{n}\right)^{2}} .
$$

Update $\omega_{k}$ :

$$
\omega_{k}^{n+1} \leftarrow \frac{\int_{0}^{\infty} \omega\left|\widehat{u}_{k}^{n+1}(\omega)\right|^{2} \mathrm{~d} \omega}{\int_{0}^{\infty}\left|\widehat{u}_{k}^{n+1}(\omega)\right|^{2} \mathrm{~d} \omega} .
$$

(4) Update $\lambda$ :

$$
\begin{aligned}
& \text { Update } \lambda \text { : } \\
& \lambda^{n+1}(\omega) \leftarrow \lambda^{n}(\omega)+\tau\left(f(\omega)-\sum_{k} \widehat{u}_{k}^{n+1}(\omega)\right) .
\end{aligned}
$$

(5) Repeat step (2) (4) until the convergence $\sum_{k}\left\|\widehat{u}_{k}^{n+1}-\widehat{u}_{k}^{n}\right\|_{2}^{2} /\left\|\widehat{u}_{n}^{k}\right\|_{2}^{2}<\varepsilon$. At the end of the cycle, $K$ components are output.

2.2. Compressive Sensing. If a sparse signal or the signal is sparse in a transform domain, the original signal can be recovered with high precision through the signal of a small number of samples [13].

If an original signal can be expanded under an orthogonal base, it can be shown as

$$
x=\sum_{i=1}^{N} \alpha_{i} \Psi_{i}
$$

where $x \in R^{N \times 1}$ denotes the original signal, $\Psi=\left[\Psi_{1}, \Psi_{2}, \ldots, \Psi_{N}\right] \in R^{N \times N}$ represents the transform domain, and $\alpha$ is a sparse vector representing the transform coefficient corresponding to $\Psi$. The matrix form of (6) is as follows:

$$
x=\Psi \alpha .
$$

It is assumed that the sparse vector includes $S$ nonzero (or far from zero) coefficients and $S$ is much less than $N$, and $x$ is $S$-sparse. $S$ is the sparsity of $x$ in the orthogonal base.

$x$ can be projected from high dimension onto low dimension, and a compression signal $y$ can be obtained:

$$
y=\Phi x,
$$

where $\Phi$ is $M \times N$-dimensional measurement matrix and $M$ is much less than $N$. $N \times 1$-dimensional signal can be downsampled to $M \times 1$ dimension according to (8).

Equation (7) is substituted into (8), and then (9) can be obtained:

$$
y=\Phi x=\Phi \Psi \alpha=A \alpha,
$$

where $A=\Phi \Psi \in R^{M \times N}$ is a sensing matrix.

As shown in (9), it is a question of solving the linear equation set to recover $x$ from compressed signal $y . \alpha$ can be obtained by solving (9), and $x$ will be known from (7). The solution of (9) can be expressed as

$$
\left.\begin{array}{ll}
\arg \min & \|\alpha\|_{0} \\
\text { s.t. } & y=A \alpha
\end{array}\right\},
$$

where $\|\alpha\|_{0}$ is 0 -norm of $\alpha$.

As dimension $M$ of $y$ is much smaller than the total number of samples N, the problem described by (10) is NPhard. Several feasible theoretical solutions for $\alpha$ may exist, so there is not fast solution of the equation set. In this case, an approximate solution is required. The 0 -norm in (10) can be transformed into 1-norm to obtain an accurate sparse solution of $\alpha$, as shown in

$$
\left.\begin{array}{ll}
\arg \min & \|\alpha\|_{1} \\
\text { s.t. } & y=A \alpha
\end{array}\right\} .
$$

The sparse vector $\alpha$ can be solved with little error, but the prerequisite is that $A$ meets the RIP criterion as follows:

$$
(1-\delta)\|\alpha\|_{2}^{2} \leq\|A \alpha\|_{2}^{2} \leq(1+\delta)\|\alpha\|_{2}^{2},
$$

where $\delta \in(0,1)$. That is, the measurement matrix $\Phi$ is not related to the sparse dictionary $\Psi$.

\section{The Proposed Feature Extraction Scheme}

Figure 1 explains the method proposed in this paper and its principles. The steps of this method are listed as follows.

\subsection{Signal Preprocessing}

3.1.1. Order Tracking. Referring to order tracking steps [11], the vibration signal of rolling bearings $x(t)$ is pretreated, by which the time-domain nonstationary signal $x(n)$ can be converted to the angle-domain stationary signal $x(\theta)$ under variable working conditions.

Since the Fourier dictionary is chosen in the following as an orthogonal dictionary, the input signal length of 


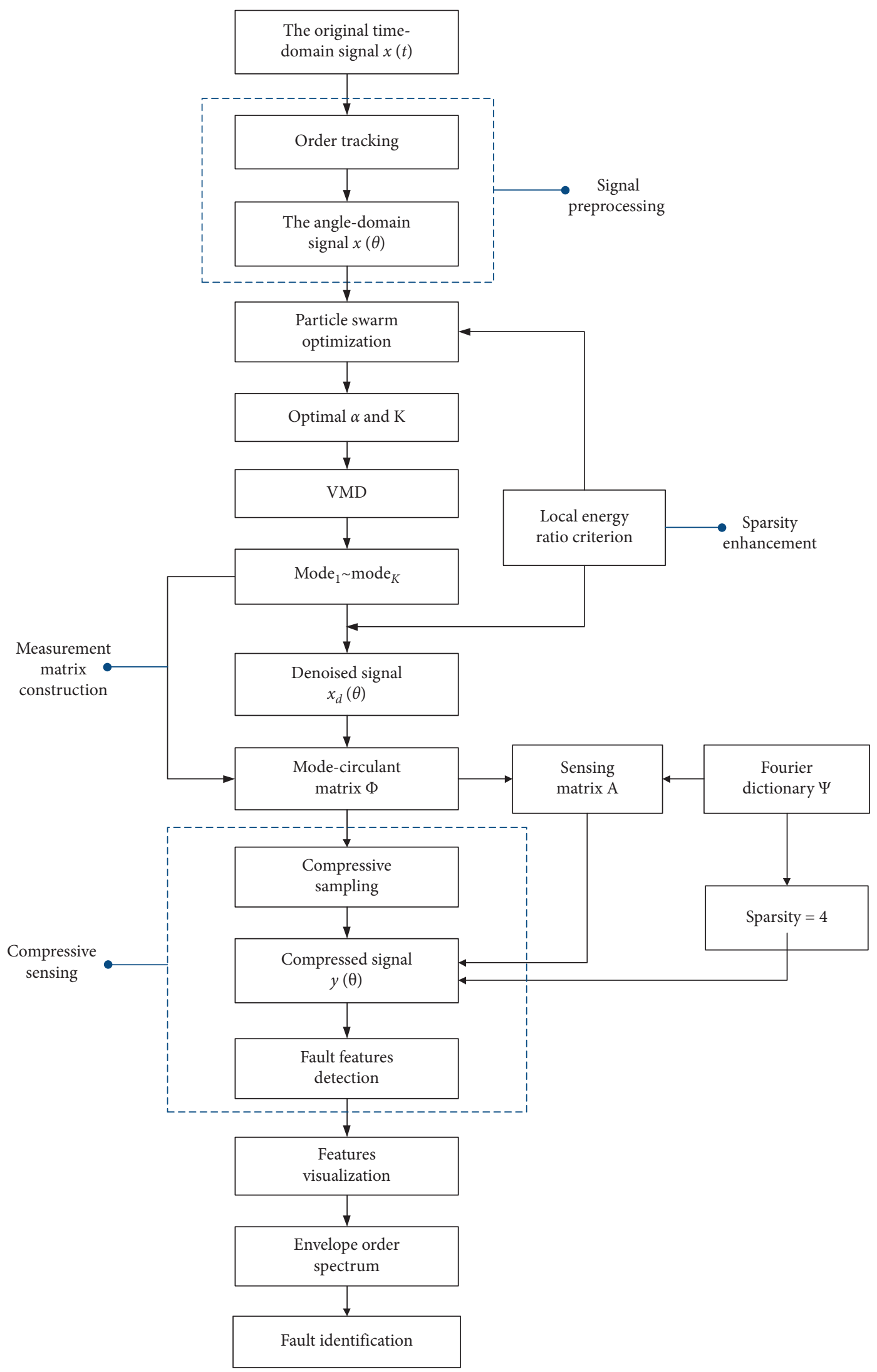

FIGURE 1: Schematic of the proposed bearing weak fault feature extraction method. 
compressed sensing needs to match the processing capacity of the computer. When it does not fit well, order tracking may have also additional use of compressing signals without loss of bearing fault feature.

\subsection{Signal Decomposition}

3.2.1. Optimization of VMD Parameters. Before the vibration signal is decomposed, we should predefine the balancing parameter of the data-fidelity constraint $\alpha$ and the number of the modes $K$. As a type of intelligence optimization algorithm, Particle Swarm Optimization (PSO) can fully consider the interaction between two parameters and find the global optimal solution without traversing all the parameter groups in the interval. Therefore, this paper uses PSO to optimize the parameters of VMD in parallel, which can automatically select the best combination of parameters, avoiding the interference of human subjective factors. Therefore, $x(\theta)$ is the input of the PSO algorithm, and the energy ratio $\left(E_{\mathrm{SN}}\right)$ is used as a basis for quantitative comparison in this paper. $E_{\mathrm{SN}}$ represents the ratio of energy generated in the case of bearing component failure to the total energy of vibration, and its definition is as follows:

$$
E_{\mathrm{SN}}=\frac{\left|X\left(k_{i t}\right)\right|^{2}+\left|X\left(k_{\mathrm{ot}}\right)\right|^{2}+\left|X\left(k_{\mathrm{bt}}\right)\right|^{2}+\left|X\left(k_{\mathrm{ct}}\right)\right|^{2}}{\sum_{i=1}^{N}|X(i)|^{2}},
$$

where $N$ is the length of the signal to be analyzed and $X(i)$ is the amplitude of the envelope order spectrum. $X\left(k_{\mathrm{it}}\right), X\left(k_{\mathrm{ot}}\right), X\left(k_{\mathrm{bt}}\right)$, and $X\left(k_{\mathrm{ct}}\right)$ represent the energy of the characteristic order of bearing outer race, inner race, rolling element, and cage fault, respectively, and $k_{\mathrm{it}}, k_{\mathrm{ot}}, k_{\mathrm{bt}}$, and $k_{\mathrm{ct}}$, respectively, denote the serial number corresponding to the fault characteristic order of outer race, inner race, rolling element, and cage.
3.2.2. Signal Decomposition with VMD. After the parameters are optimized, according to (1)-(5), $x(\theta)$ is decomposed by VMD to obtain a series of modes, mode $1 \sim$ mode $K$. These modes are involved for two uses: (1) The main modes (if mode $i$ ) will be obtained by comparing $E_{\mathrm{SN}}$ of different modes for noise reduction and sparsity enhancement. If mode $i$ $(i=1,2, \ldots, K)$ is the mode with the maximum $E_{\mathrm{SN}}$ value, then it is the main mode. Later mode $i$, also called $x_{d}(\theta)$, will take the place of $x(\theta)$ to participate in further compressive sensing. (2) Mode $1 \sim$ mode $K$ will be used to construct the measurement matrix needed in CS, as detailed below.

3.3. MCM Construction. In this paper, the measurement matrix, called the mode-circulant matrix (MCM), is constructed by the modes decomposition of VMD, as described in the following.

As is shown in (14), the mode matrix $I$ can be expressed by mode $1 \sim$ mode K. $n$ is the length of $x(\theta)$. The mode $i$ is $\left(a_{1}^{i}, a_{2}^{i}, \ldots, \quad a_{n}^{i}\right)$, and the superscript $i$ represents the $i$ th mode function:

$I=\left[\begin{array}{c}\bmod e_{1} \\ \bmod _{2} \\ \vdots \\ \bmod e_{i} \\ \vdots \\ \bmod _{K}\end{array}\right]=\left[\begin{array}{ccccc}a_{1}^{1} & a_{2}^{1} & \ldots & a_{n-1}^{1} & a_{n}^{1} \\ a_{1}^{2} & a_{2}^{2} & \ldots & a_{n-1}^{2} & a_{n}^{2} \\ \vdots & \vdots & \vdots & \vdots & \vdots \\ a_{1}^{i} & a_{2}^{i} & \cdots & a_{n-1}^{i} & a_{n}^{i} \\ \vdots & \vdots & \vdots & \vdots & \vdots \\ a_{1}^{K} & a_{2}^{K} & \cdots & a_{n-1}^{K} & a_{n}^{K}\end{array}\right]=\left[h_{1}, h_{2}, h_{3}, \ldots, h_{n}\right]$

The measurement matrix $\Phi$ can be acquired by circulating the mode matrix $I n_{c}$ times [29], as shown in (15). $\Phi$ is the MCM. $n_{c}$ is the number of cycles:

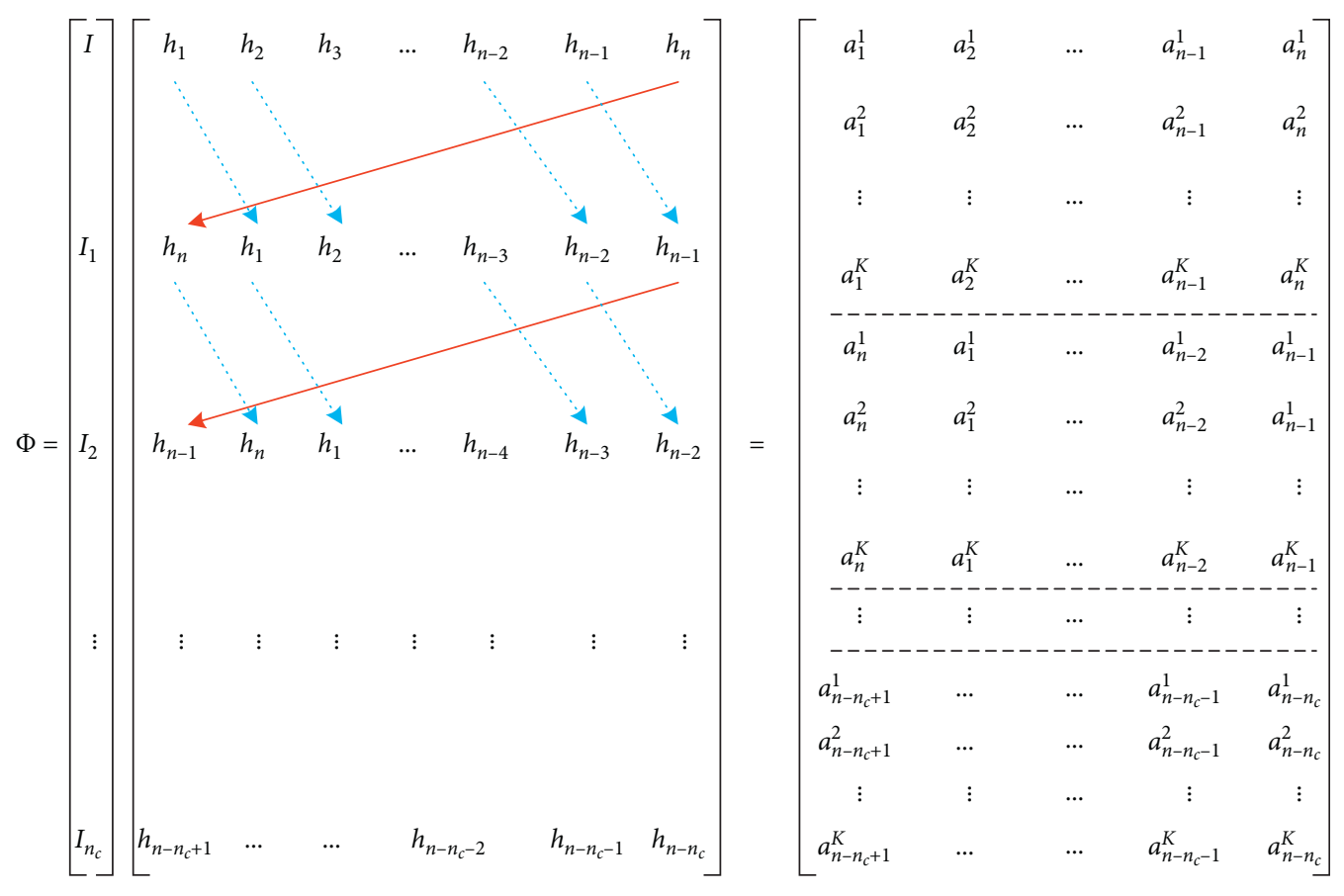


The number of rows of $\Phi$ can be calculated according to (16), that is, the number of measurements $m$ :

$$
m=\left(n_{c}+1\right) \times K \text {. }
$$

\subsection{Compressive Sensing}

3.4.1. Compressive Sampling. According to (8), the MCM is utilized to reduce the dimensions of $x(\theta)$, which compresses the $\mathrm{N}$-dimensional signal $x(\theta)$ into M-dimensional $y(\theta)$.

3.4.2. Transformation Selection. In this study, the Fourier dictionary is selected for a sparse representation of signals. The reason is that not only is the VMD mode of bearing fault signal sparse in the Fourier domain, but also the sparsity of harmonic signal in the Fourier domain is fixed $[12,13,21,30]$, which is very beneficial to fault feature detection. To investigate the properties of the Fourier dictionary, the inner race fault signal of a rolling bearing was simulated in Section 4.

3.4.3. Fault Feature Detection. The Fourier dictionary $\Psi$ and the MCM $\Phi$ make up the sensing matrix A. According to (11), the sparse vector $\alpha$ can be detected from the compressed signal $y(\theta)$ by orthogonal match pursuit with the sensing matrix A and sparsity. The sparsity setting should be taken into account in the study.

It is known that the typical fault features of rolling bearings are that the fault impact excites resonance of natural frequency, and this frequency is modulated by faulty feature frequency as the carrier frequency. Thus, when the natural frequency and feature frequency (sideband frequency) are detected simultaneously, that is, sparsity is set to 4 , the faulty feature frequency can be extracted.

\subsection{Fault Diagnosis of Rolling Bearings}

3.5.1. Feature Visualization. After the crucial frequencies detected, the signal recovery can be achieved with the Fourier dictionary $\Psi$ and the sparse vector $\alpha(\theta)$ using (7). Due to the amplitude modulation of the recovered signal, the envelope demodulation should be applied with Hilbert transform. Then the envelope spectrum is obtained by the Fourier transform of the envelope signal to realize feature extraction.

3.5.2. Fault Identification. Fault diagnosis for rolling bearings can be achieved by comparing the dominant components in the envelope spectrum with the calculation value of the fault characteristic frequency of the bearing.

\section{Simulation}

To verify the properties of the Fourier dictionary and MCM in this method, the fault signal of the inner race of the rolling bearing is constructed under speed-up conditions. The simulations are as follows [31, 32].
4.1. Rolling Bearings Fault Signal Modeling. The simulated signal of rolling bearings' fault model can be expressed strictly as follows:

$$
x(t)=\sum_{i=1}^{N} A_{i} s\left(t-T_{i}-\tau_{i}\right),
$$

where $A_{i}$ is the amplitude of the $i$ th impulse and $s\left(t-T_{i}-\tau_{i}\right)$ is the $i$ th impulse oscillation generated by the fault with the natural frequency $f_{n}$. Generally, $s(t)$ is an exponential decay process. Under variable speed conditions, $s(t)$ and $A_{i}$ can be refined into

$$
\begin{aligned}
s(t) & =e^{-B\left(t-T_{i}-\tau_{i}\right)} \cdot \sin \left(2 \pi f_{n}\left(t-T_{i}-\tau_{i}\right)\right), \\
A_{i} & =A \cos \left(2 \pi f_{r} t+\varphi_{A}\right)+C_{A} .
\end{aligned}
$$

The bearing fault simulation signal can be concluded as follows:

$$
\begin{aligned}
x(t)= & \sum_{i=1}^{N}\left(A \cos \left(2 \pi f_{m} t+\varphi_{A}\right)+C_{A}\right) \\
& \cdot e^{-B\left(t-T_{i}-\tau_{i}\right)} \cos \left(2 \pi f_{n}\left(t-T_{i}-\tau_{i}\right)\right),
\end{aligned}
$$

where $B$ is an attenuation coefficient, $T_{i}$ is the corresponding time or the $i$ th fault impulse, $\tau_{i}=\lambda_{i} \cdot T_{i}$ is the minor random fluctuation around $T_{i}$, and $n(t)$ represents random noise.

The calculation process of $T_{i}$ under variable speed condition is as follows.

In this simulation, the fault characteristic order of bearing inner race is $O_{i}=14.9$; that is, the fault impact caused by the rotor every rotation cycle is 14.9. Also, it is assumed that the variation equation of rotor speed is $f_{r}(t)=a t+b$. The relationship between the rotating angle of the inner race and the time coordinate corresponding to the fault impulse can be expressed by

$$
\int_{0}^{t} f_{r}(t) \mathrm{d} t=\frac{a}{2} t^{2}+b t=\theta_{i}
$$

where $\theta_{i}=i / O_{i}(i=1,2, \ldots, K)$. The time coordinate $T_{i}$ corresponding to the impact can be obtained by solving the above equation.

This study assumes $A=2, C_{A}=0.5, \Phi_{A}=30^{\circ}, B=800$, $f_{n}=3000, \lambda_{i}=0.01, a=4, b=15$, and $O_{i}=14.9$. Besides, the fault characteristic orders of bearing inner race, rolling elements, and cage are 9.92, 2.4, and 0.4, respectively. In this paper, the sampling frequency of the simulation signal is $12.8 \mathrm{kHz}$, and the sampling length is 8192 . The simulation signal of inner race defect without noise is shown, respectively, in Figure 2.

The fault vibration signal of rolling bearing measured $x_{b}(t)$ is composed of the fault signal $x(t)$ and some random noise $n(t)$. It can be expressed as follows:

$$
x_{b}(t)=x(t)+n(t) .
$$

Gaussian noise is added to the simulated signal in Section 4.1 to hide the fault features in the noise. To quantify the added noise influence, two means of calculating signalto-noise ratio (SNR) are used, one for gauging the local peaks 


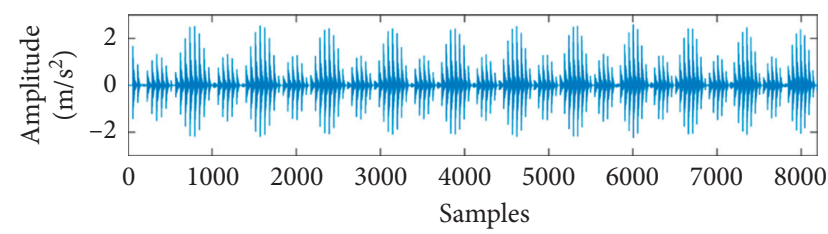

Figure 2: The inner race fault simulation signal.

of the random noise (referred to as SNR1) [33] and another for further gauging the noise level across fault characteristic order (referred to as SNR2) [34]. SNR1 is defined as

$$
\mathrm{SNR} 1=20 \log _{10}\left(\frac{P_{s}}{A_{n}}\right),
$$

where $P_{s}$ denotes the RMS value of the signal $x(t)$ and $A_{n}$ represents the peak value of the noise. According to (23), the SNR1 of the fault simulation signal $x_{b}(t)$ is $-22.7 \mathrm{~dB}$.

SNR2 is defined as follows:

$$
\begin{aligned}
\mathrm{SNR} & =10 * \log _{10}\left(\frac{S\left(O_{t}\right)}{N\left(O_{t}\right)}\right), \\
S\left(O_{t}\right) & =S\left(O_{1 t}\right)+S\left(O_{2 t}\right)+\cdots+S\left(O_{\mathrm{it}}\right)+\cdots+S\left(O_{\mathrm{nt}}\right), \\
n & =\left\lceil\frac{O_{\max }}{O_{t}}\right\rceil \\
S\left(O_{i t}\right) & =\sum_{j=k_{i t}-l}^{k_{i t}+l}|X(j)|^{2}, \quad i=[1, n], l \neq 0, \\
N\left(O_{t}\right) & =\sum|X(j)|^{2}-S\left(O_{t}\right),
\end{aligned}
$$

where $O_{\max }$ is the maximum order to be analyzed and $X(j)$ is the amplitude of the envelope order spectrum. $S\left(O_{t}\right)$ represents the total energy of bearing fault characteristic order and its multiple in the range of 0 to $O_{\max } . S\left(O_{i t}\right)$ is the energy which is in the vicinity of $i O_{t}-l \Delta o$ and $i O_{t}+l \Delta o$ and it centers on $i O_{t}$. $O_{i t}$ is the $i$ time of theoretical fault characteristic order, and $k_{i t}$ is the serial number of $O_{i t} . N\left(O_{t}\right)$ denotes the energy of noise in the same range and it excludes $S\left(O_{t}\right)$. According to (24), the SNR2 of the fault simulation signal is $x_{b}(t)-16.25 \mathrm{~dB}$.

The signal after being affected by noise is shown in Figure 3(a). Additionally, Figure 3(b) provides the corresponding speed trend.

4.2. Optimization of VMD Parameters. According to the steps of the proposed method in Section 3, the signals Figure 3(a) are resampled in the angle domain, respectively, and the obtained angular waveform is as shown in Figure 4.

Particle Swarm Optimization has been applied to analyze the noisy signal in angle domain. A fitness curve can be obtained, as being presented in Figure 5. The setting of the main parameters and optimization results is given in Table 1. The maximum value of the local energy ratio appears at
0.1475 in the tenth generation, and the best combination of decomposition parameters is $[\alpha, K]=[664.7,6]$.

Subsequently, the modes are generated by the angular signal's VMD decomposition in which $K=6$ and $\alpha=664.7$ are used. Table 2 shows the fitness values for each mode, and mode 4 has the maximum fitness value, almost 3 5 times that of other modes. So the mode 4 is the main mode, that is, denoised signal for the simulation signal with noise.

4.3. Properties Verification. Based on Section 4.2, this part will further validate three properties: (1) after noise reduction of rolling bearing fault signal, the sparsity is promoted in Fourier dictionary; (2) the sensing efficiency of MCM is higher than that of Gaussian random matrix (GRM); (3) MCM-based CS (MCM-CS) is more effective than Narrowband Envelope Analysis (NEA) in the feature extraction of bearing fault.

4.3.1. Sparsity Analysis of Bearing Fault Signal. In the sparse transform domain, only $K$ nonzero elements are sparse (where $K$ is far less than the signal length). Generally, the signal does not necessarily satisfy the above requirement of absolute sparsity. The signal can be regarded as an approximate sparse signal when the sparse transform coefficients of the signal decrease exponentially to zero $[12,13,30]$. Therefore, we can judge whether the rolling bearing fault signal in 4.1 is sparse under the Fourier dictionary by analyzing the decreasing trend of Fourier coefficients.

In this paper, firstly, the exponential function is utilized to fit the attenuation curve. Secondly, we calculate the sum of squares error (SSE) and coefficient of determination (Rsquared) between the original curve and the fitted curve to assess whether the attenuation of the sparse coefficient is close to the exponential attenuation. Here, SSE is the square sum of the errors of the corresponding points of the fitting data and the original data. R-square is used to represent the quality of the fitting by the change of data. The closer it is to 1 , the better the data fitting is. The closer SSE is to 0 , the more successful the fitting is.

The angular waveform without noise is shown in Figure 4 (a), and the sparse coefficients obtained by the Fourier transform are shown in Figure 6(a). The absolute values of Fourier transform coefficients are sorted from large to small, and the sorted result is shown in Figure 6(b).

The attenuation curve of Figure 6(b) is fitted by using the least-square method, through the curve fitting toolbox of MATLAB, and the formula obtained is $f(x)=0.2 e^{-0.059 x}+0.03 e^{-0.0018 x}$. The actual attenuation curve and the fitting curve are as presented in Figure 7, where SSE is 0.011 and $R$-square is 0.984 , which are both close to the ideal value. Furthermore, from the detailed area, it is obvious that the sparse coefficients decrease to zero. So it can be considered that the Fourier coefficients decay to zero exponentially, indicating that the vibration signal of rolling bearing is almost sparse in the Fourier domain.

The existence of noise in the vibration signal will increase the difficulty of sparse representation in the Fourier domain. 


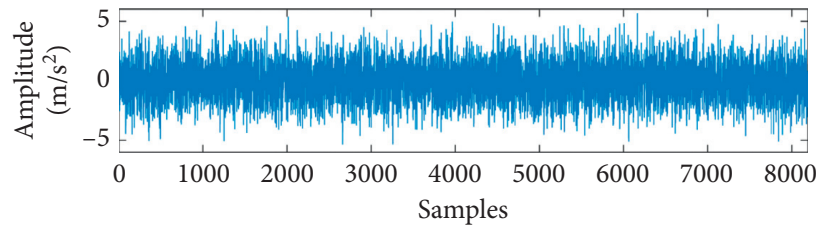

(a)

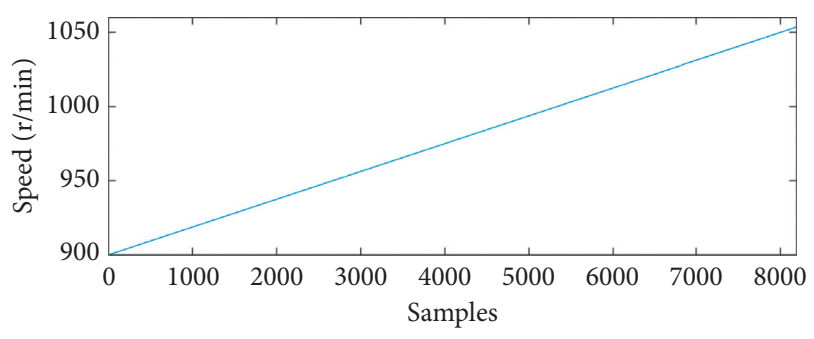

(b)

FIGURE 3: Simulated signal of bearing fault with noise. (a) The waveform of vibration and (b) speed trend.

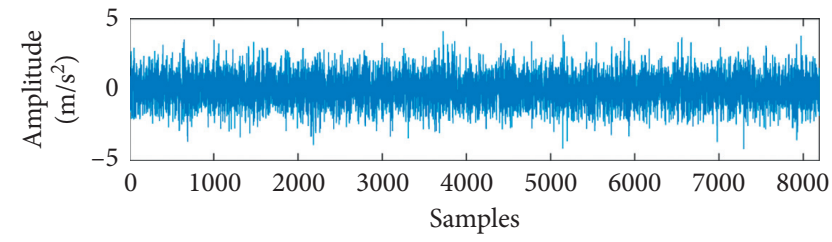

Figure 4: The angular signal with noise.

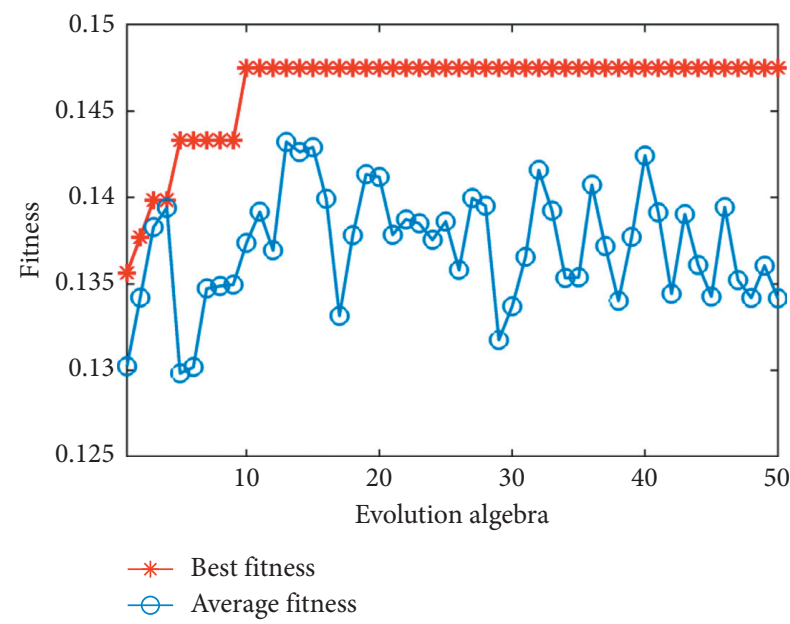

FIgURE 5: The trend of local characteristic order amplitude with evolution algebra.

TABLE 1: Parameters setting and optimization results of PSO.

\begin{tabular}{lccc}
\hline Parameters & $\alpha$ & & $K$ \\
\hline Range of values & {$[0,2000]$} & & {$[1,10]$} \\
Maximum evolution & & 50 & \\
Maximum population & & 10 & \\
Local search speed & & 1 & \\
Global search speed & 1.2 & \\
Optimization results & 664.7 & & 6 \\
\hline
\end{tabular}

Sparse coefficients of the noisy signal and the sequencing result are shown in Figures 8(a) and 8(b), respectively. The fitting result of the attenuation curve is shown in Figure 9, and the formula of the fitted curve is $f(x)=0.065 e^{-0.00047 x}-5.5 \times 10^{-12} e^{-0.0052 x}$. Due to the influence of noise, SSE is as high as 0.18 and R-square is only 0.852 , both of which deviate slightly from the ideal value. What is more, the attenuation curvature of sparse coefficients of the noisy signal is increasing, not decreasing as exponential attenuation. Thus, sparse coefficients in Figure 8(b) cannot be concluded to attenuate to zero exponentially, which means the polluted signal has inadequate sparsity affected by full-band noise.

Finally, the sparsity of mode 4 in Table 2 is analyzed in the Fourier domain. Sparse coefficients of the denoised signal and the sorting result are shown in Figures 10(a) and 10(b), respectively. The attenuation curve of Figure 10(b) is further fitted, and the formula obtained is $f(x)=0.16 e^{-0.091 x}+0.056 e^{-0.0031 x}$. In Figure 11, SSE is $0.007, \mathrm{R}$-square is 0.994 , and the actual attenuation curve almost overlaps the fitted curve. It can be inferred that the sparse coefficients of the denoised signal can be decreased exponentially to zero under the Fourier transform. Compared with the noisy signal, the sparsity of the denoised signal is greatly enhanced.

4.3.2. High Sensing Efficiency of MCM. Gaussian random matrix (GRM) is frequently used as a measurement matrix of CS for a typical one-dimensional vibration signal [18-21, 25]. The reason is just simple and convenient to construct, and most importantly, the measurement matrix constructed by the basis vector obeying the Gauss distribution can be roughly guaranteed to be irrelevant to any sparse basis.

In this study, a new compressive sensing measurement matrix is constructed in the form of the circulant matrix by using the VMD modes. Its sensing efficiency is much higher than GRM, which means the extraction accuracy of the MCM can be achieved by fewer measurements than GRM. The following parts will demonstrate it.

This verification firstly used the modes in Table 2 to construct the MCM and then applied it and GRM, respectively, to compress the denoised vibration signal. Fault feature extraction is considered successful when the fault feature order appears in the final envelope spectrum.

Figure 12(a) and 12(b) are the final envelope order spectra obtained by using MCM and GRM, respectively, when the number of measurements is 600 . The dominant order in Figure 12(a) is consistent with the inner simulated fault order $O=14.9$, representing a $100 \%$ success rate of fault feature extraction, while Figure 12(b) fails; that is, the success rate is 0 . According to (24), the SNR2 of Figure 12(a) and $12(\mathrm{~b})$ are $33.4 \mathrm{~dB}$ and $-48.7 \mathrm{~dB}$, respectively, and bearing fault can be directly identified with the former. 
TABLE 2: Fitness values obtained by analyzing simulation signal with VMD.
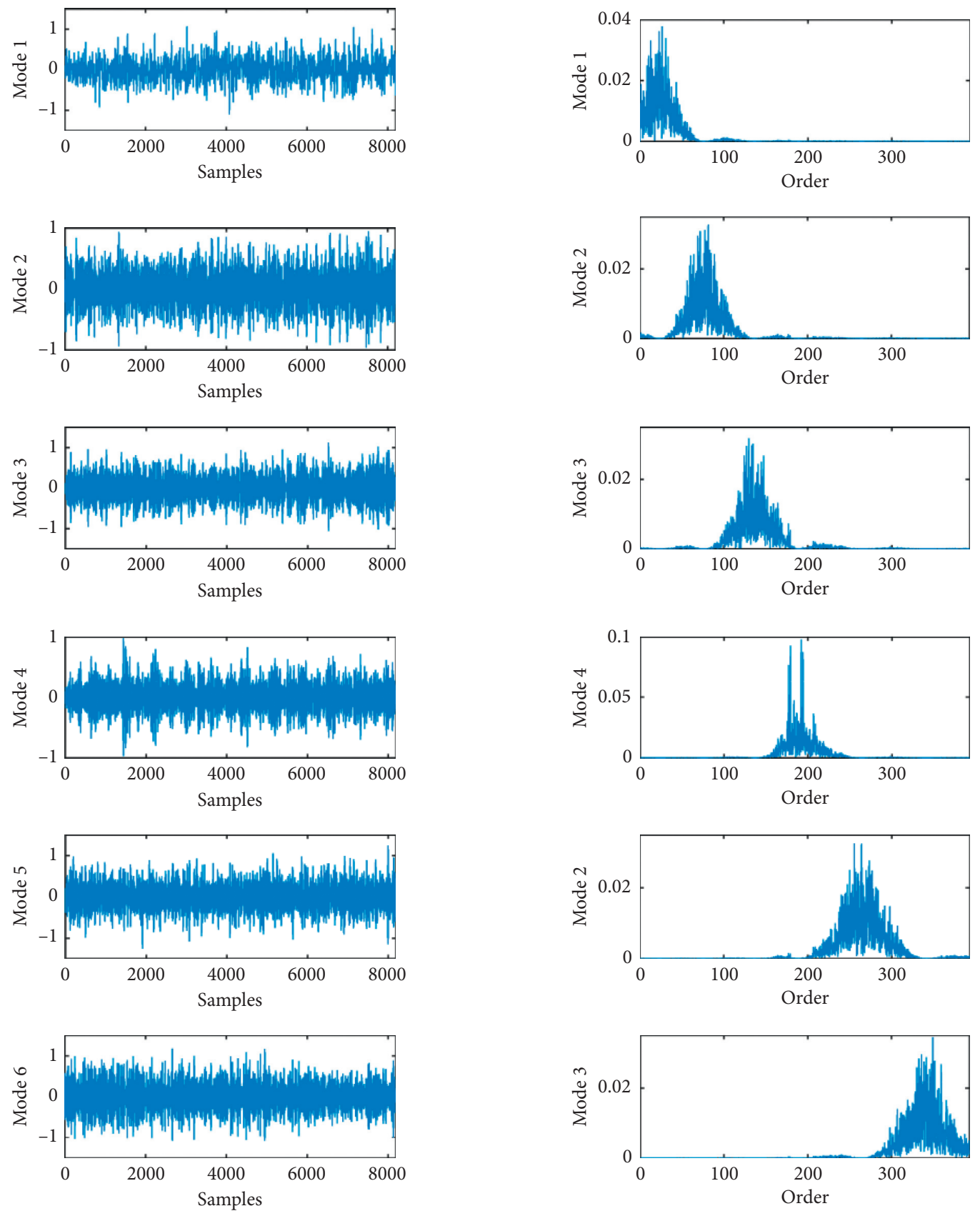

Moreover, the change in success rate is shown in Figure 13 as the number of measurements increases from 20 to 1000. From Figure 13, when the number of measurements is only 60, the MCM can successfully extract bearing fault features, and the results are stable and consistent within a varied number of measurements. By contrast, the GRM has obvious randomness and poor robustness although it can occasionally extract fault features under certain measurements. Therefore, sensing efficiency of the MCM is higher than that of the GRM, which means that the beneficial information of the signal can be measured more effectively.

4.3.3. Validation of NEA Method. Section 4.3.2 has verified that MCM has higher sensing efficiency than GRM. Combined with CS, MCM-CS can effectively extract bearing fault features under a fewer number of measurements. To further highlight the significance of MCM-CS for bearing fault 


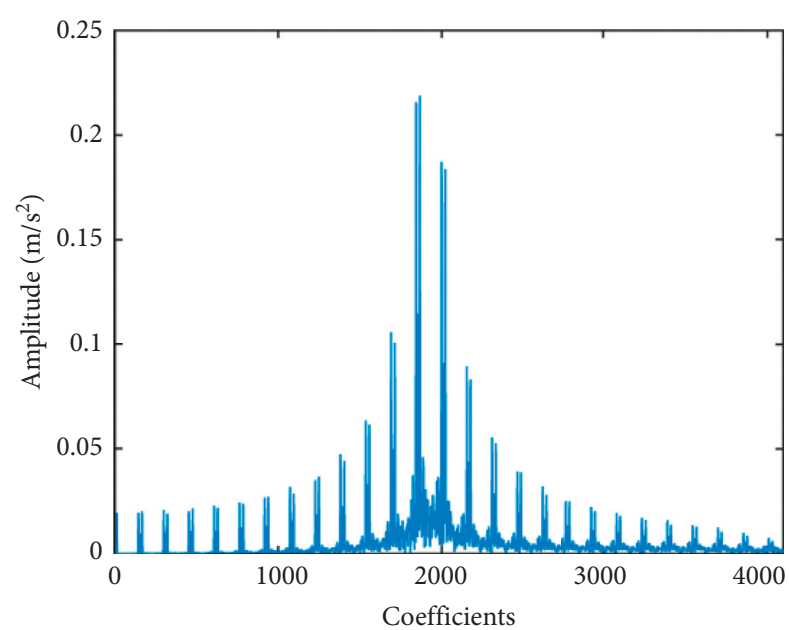

(a)

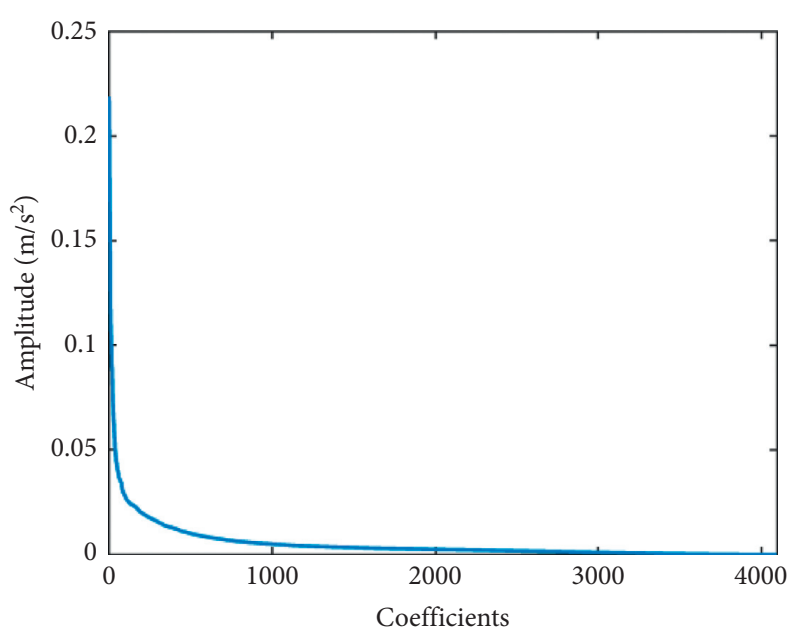

(b)

FIgURE 6: In Fourier dictionary, (a) sparse coefficients of pure simulation signal; (b) attenuation curve of (a).

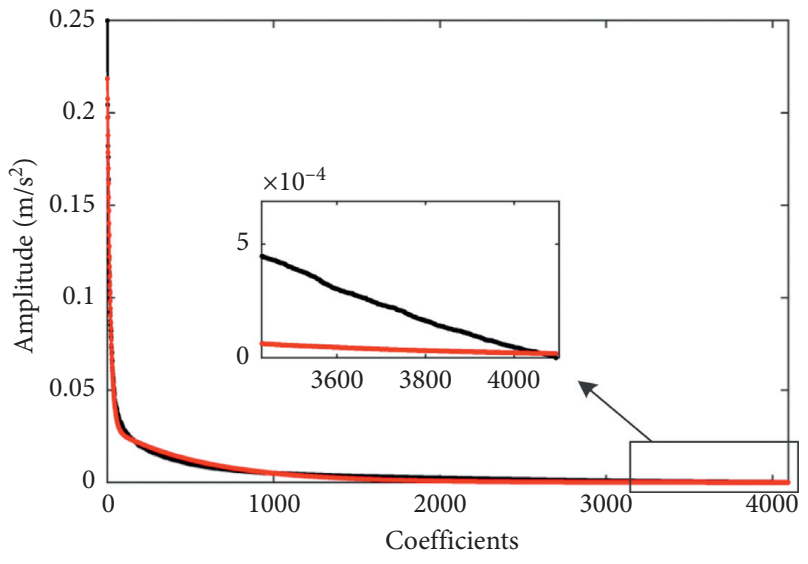

- The actual attenuation curve

$\rightarrow$ The fitted attenuation curve

FIGURE 7: The fitting result of coefficients attenuation curve in Figure 6(b): SSE $=0.011 ; R$-square $=0.984$.

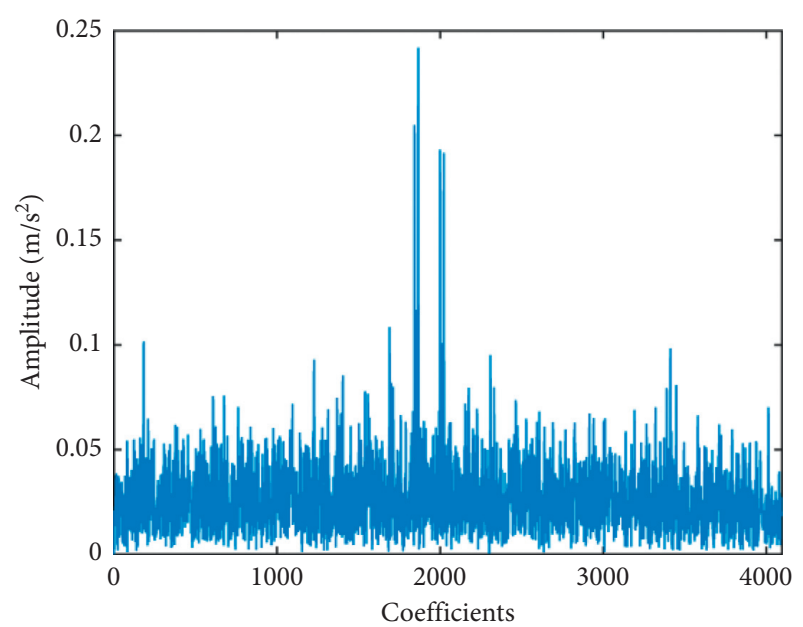

(a)

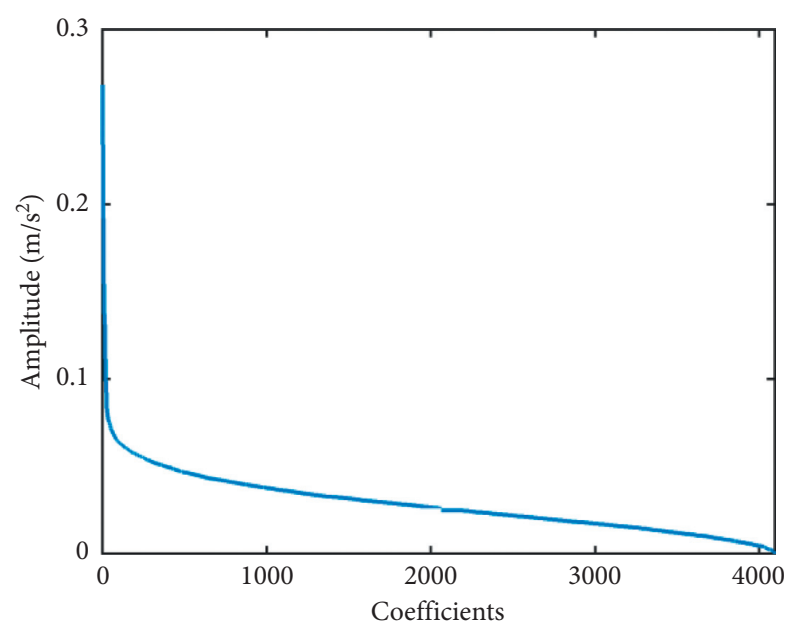

(b)

Figure 8: In Fourier dictionary, (a) sparse coefficients of the noisy signal; (b) attenuation curve of (a). 


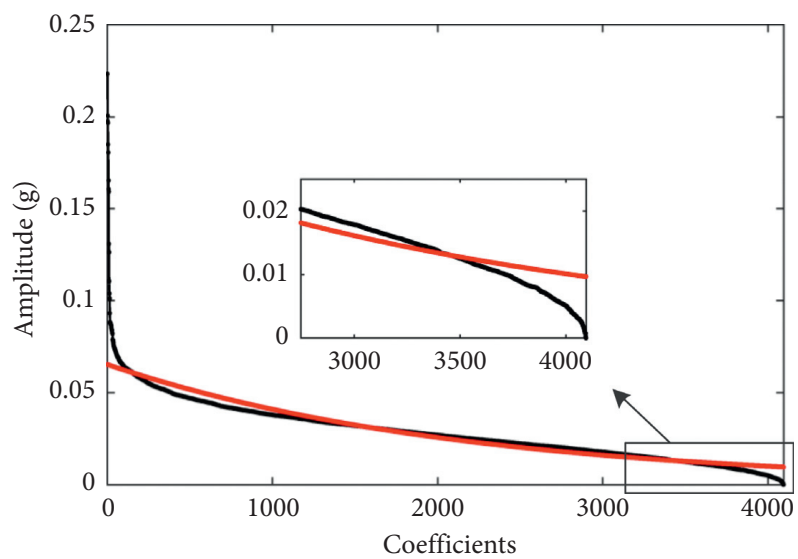

- The actual attenuation curve

$\rightarrow$ The fitted attenuation curve

Figure 9: The fitting result of coefficients attenuation curve in Figure $8(\mathrm{~b})$ : SSE $=0.18 ; R$-square $=0.852$.

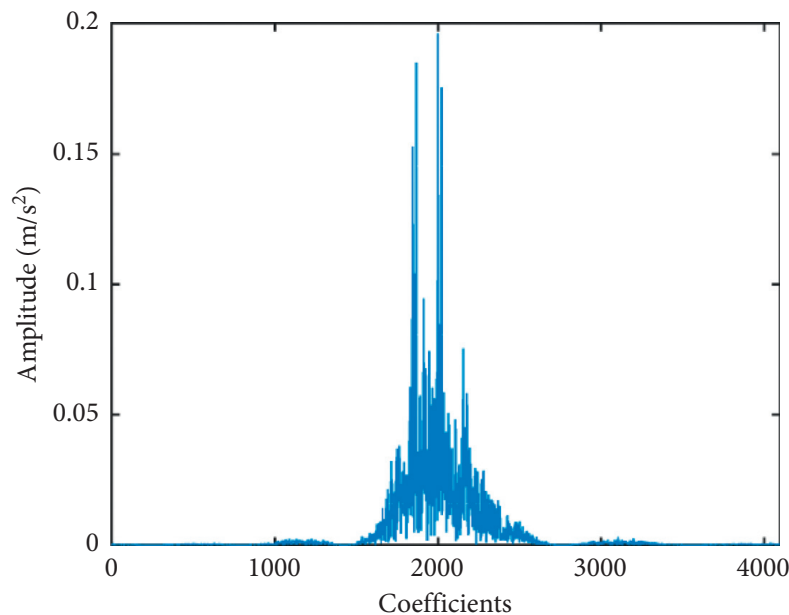

(a)

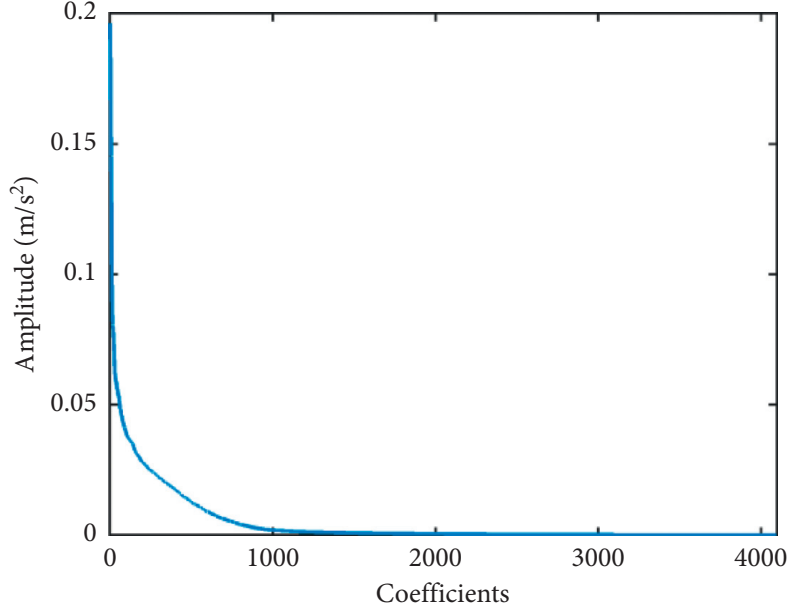

(b)

Figure 10: In Fourier dictionary, (a) sparse coefficients of the mode 3 and (b) attenuation curve of (a).

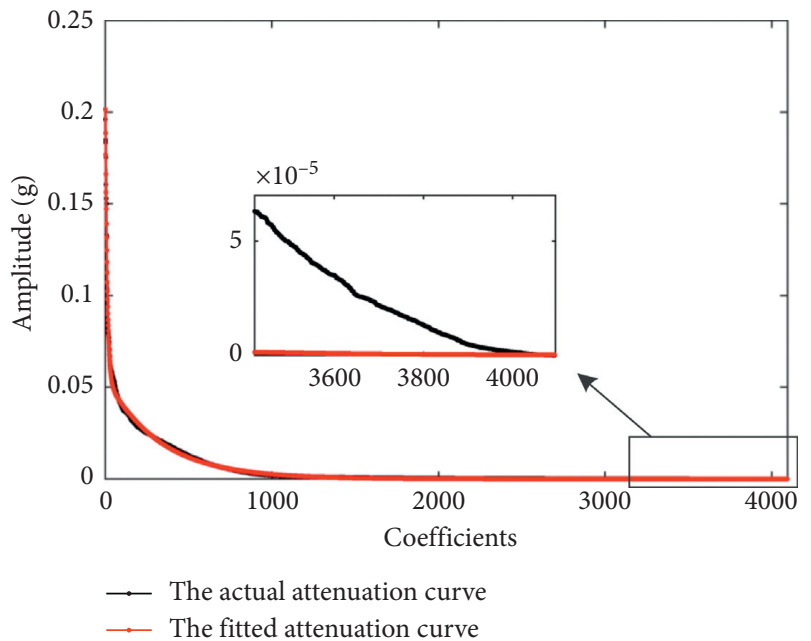

FIgURE 11: The fitting result of coefficient attenuation curve in Figure 10(b): SSE=0.007; R-square =0.994. 


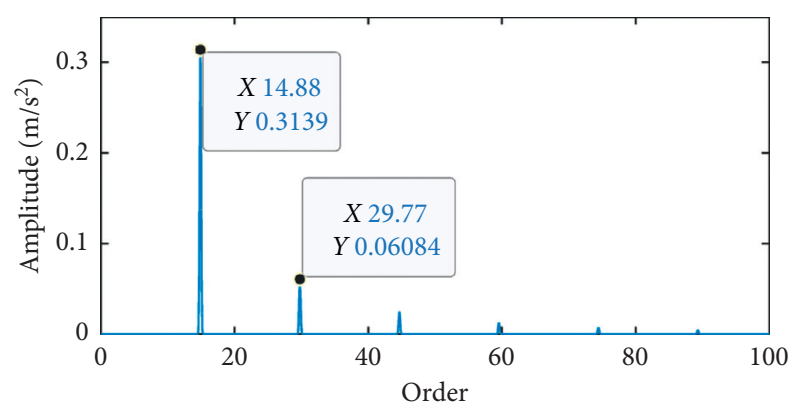

(a)

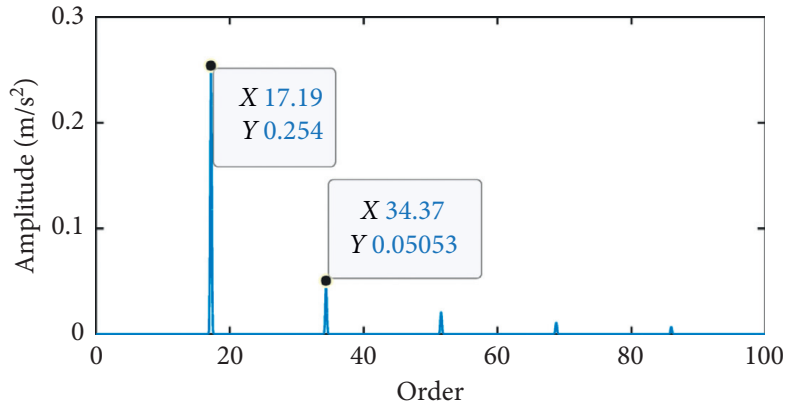

(b)

FIGURE 12: The envelope order spectra obtained by using (a) MCM: the spectrum is clear, and SNR2 is $33.4 \mathrm{~dB}$; (b) GRM: the spectrum is clear, but SNR2 is only $-44.7 \mathrm{~dB}$.

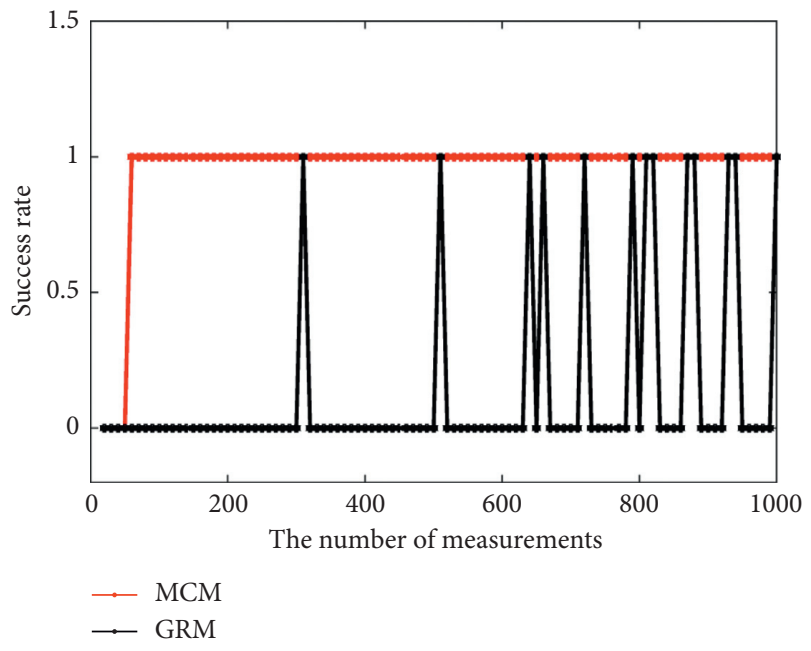

Figure 13: Comparison of detection effect of two measuring matrices.

compressive detection, the Narrowband Envelope Analysis (NEA) is selected for comparison.

Before analyzing the bearing fault signal to NEA, the most fundamental issue to be solved first is the setting of the center frequency and bandwidth of the band-pass filter. However, it fails to extract effective features based on experience frequently. In recent years, SK has been extensively used in the selection of demodulation band parameters and gained good reputation [35]. Thus, SK is used to design the band-pass filter in the NEA method in this section. Besides, considering variable speed conditions, NEA is carried out as follows:

(1) Even angle resampling in the speed domain, order tracking is applied to the original signal, achieving a stationary signal in the angle domain instead of a nonstationary signal in the time domain.

(2) The optimal envelope center frequency and bandwidth are determined by SK, based on which a bandpass filter is designed to filter the resampled signal.

(3) The envelope demodulation method based on the Hilbert transform is applied to achieve the envelope order spectrum.
Figure 14 shows the analysis results for the fault simulation signal shown in Figure 3(a) using the NEA based on SK and order tracking. Figure 14(a) is the kurtogram. The SK shows that the center frequency of the optimum demodulation band is $2933 \mathrm{~Hz}$, which is extremely near to the resonance frequency of the simulated signal. This proves that SK is highly significant. Figure 14(b) shows the obscure envelope order spectrum, where the amplitude of 2.099 order is the highest. An order of 14.79, corresponding to the inner fault, can be seen but it is not the dominant component in this spectrum.

Besides, according to (24), the SNR2 of the filtered signal is $-9.07 \mathrm{~dB}$, which is far from the recovered signal through MCM-CS. As observed in the above, although NEA is indeed simpler than MCM-CS, it is not only unable to achieve fault compressive detection but also inferior in weak fault feature extraction.

The facts of Section 4.3 prove that the VMD mode of fault vibration signals of rolling bearings can be sparsely represented in the Fourier dictionary. What is more, the MCM-enhanced compressive sensing is endowed with higher sensing efficiency. These are the necessary conditions for CS to extract fault feature of rolling bearings in Section 5 .

\section{Experiments}

To further verify the effectiveness of the weak fault feature extraction method based on the sensing-enhanced CS, the weak fault signals of the rolling bearings under variable speed conditions are collected in the test rig described below.

5.1. Experimental Setup. To simulate the weak fault of rolling elements to a large extent, the dual-rotor test rig is selected, which is designed and built to simulate the working state of the intershaft bearing on the actual double rotor equipment. As is illustrated in Figure 15, it consists of two driving motors, \#1 intershaft bearing, \#2 bearing, \#2 bearing housing, and dual rotors. In particular, \#2 bearings are fitted with thin-walled supports.

In actual dual-rotor equipment, since the inner and outer rings of intershaft bearings are rotating, there is no fixed bearing housing for the installation of sensors, which must require indirect measurement of vibration. The 


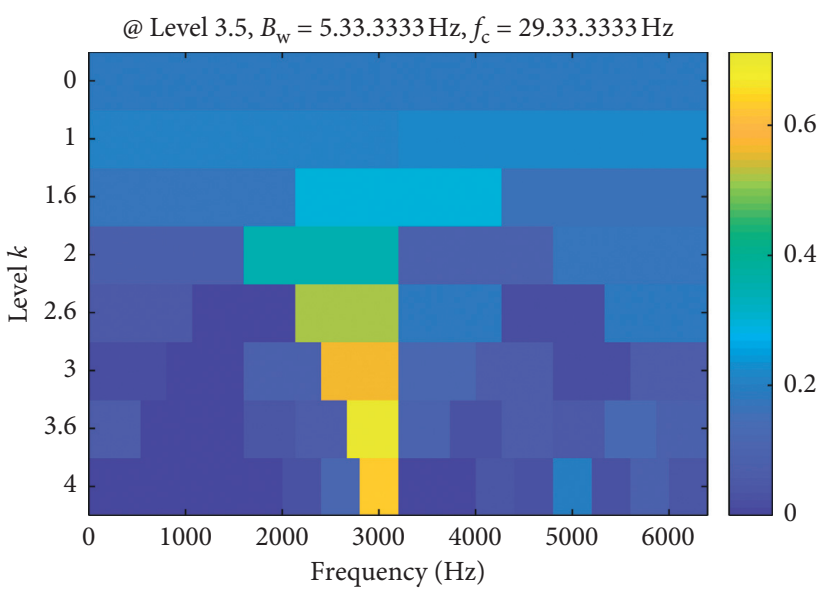

(a)

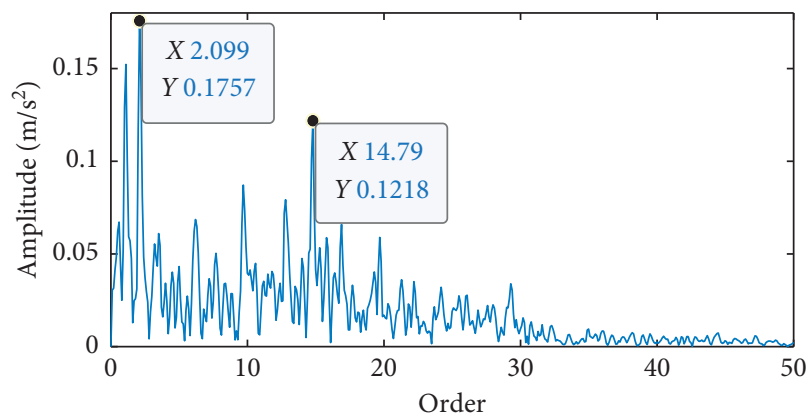

(b)

FIgURE 14: The analysis results using NEA: (a) kurtogram; (b) envelope order spectrum: the spectrum is obscure, and SNR2 is only $-9.07 \mathrm{~dB}$.

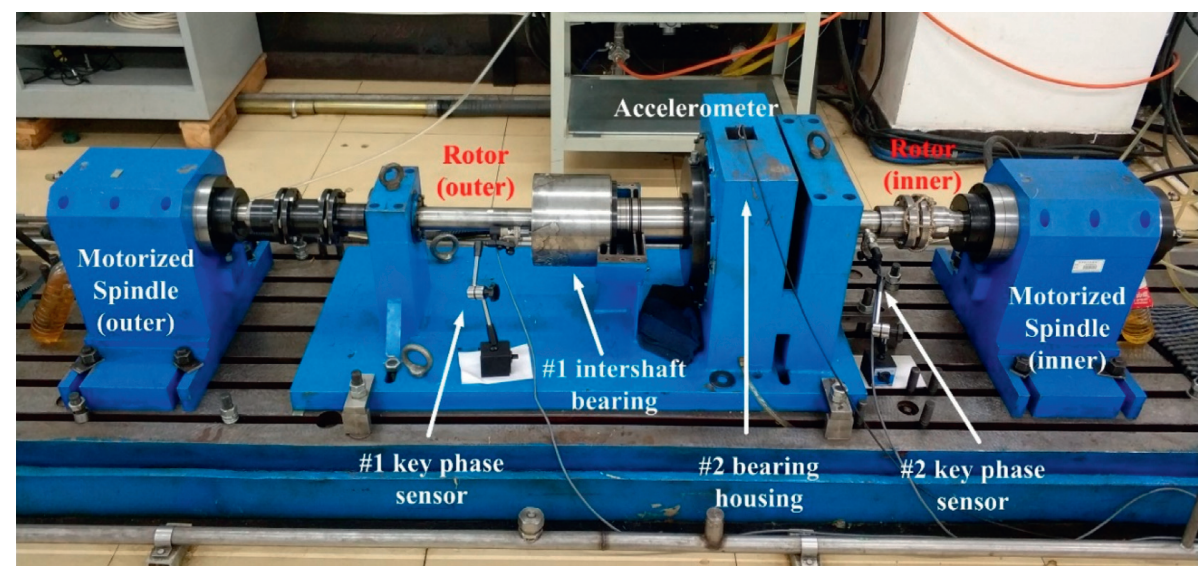

FIGURE 15: Dual-rotor vibration test rig.

indirectly measured vibration response needs to be transmitted to the adjacent bearing via the shaft and then to sensor position through the elastic support and complicated thin-wall paths so that the intershaft bearing signal is buried in the strong vibration and interference.

Similar to the actual dual-rotor equipment, \#1 intershaft bearing, in the dual-rotor test rig, has not directly linked bearing housing. Thus, although the inner and outer races of \#1 intershaft bearing are machined defectively, the accelerometer is installed at \#2 bearing housing. Figure 16 is the transfer path of vibration of \# 1 intershaft bearing in the dualrotor test rig, it can be seen that the test rig reproduces the running status of an actual intershaft bearing: the vibration response of \#1 intershaft bearing needs to be transferred to adjacent bearing via the inner rotor; then, via elastic supports and thin-walled structure, the response is transmitted to the \#2 bearing housing. The complicated transfer path indicates that the detected bearing signal is severely attenuated during the transfer. Therefore, compared with traditional rolling bearings used with a single rotor and fixed housing, the fault signal of \#1 intershaft bearing collected in this experiment is relatively weak.
The faults of inner and outer races of the intershaft bearing were simulated as follows. As for the outer race defect, a $1 \mathrm{~mm}$ deep and $1 \mathrm{~mm}$ wide groove was machined across the axis direction on the inner surface of the outer race. The outer surface of the inner race was also machined with the same size defects to simulate inner race fault.

The vibration signal is acquired by the BK4519 acceleration sensor, a key phase sensor, charge amplifier, and LMS SCADAS [36] data acquisition system. During the experiment, only the drive motor of the inner race rotor of 1 \# intermediate bearing is started, while the outer ring rotor is fixed.

The 1 \# intershaft bearing fault vibration data used in this study are divided into the following two groups: (1) outer race fault signal where the inner race speed is increased from $625 \mathrm{r} / \mathrm{min}$ to $766 \mathrm{r} / \mathrm{min}$; the sampling frequency is $25.6 \mathrm{kHz}$, and the number of vibration signals sampled for analysis is 102400; (2) inner race fault signal where rotational speed of inner race increased from $738 \mathrm{r} / \mathrm{min}$ to $825 \mathrm{r} / \mathrm{min}$; sampling frequency is $102.4 \mathrm{kHz}$, and the amount of sample is 204800 . The vibration waveforms and speed trends are shown in Figures 17 and 18. 


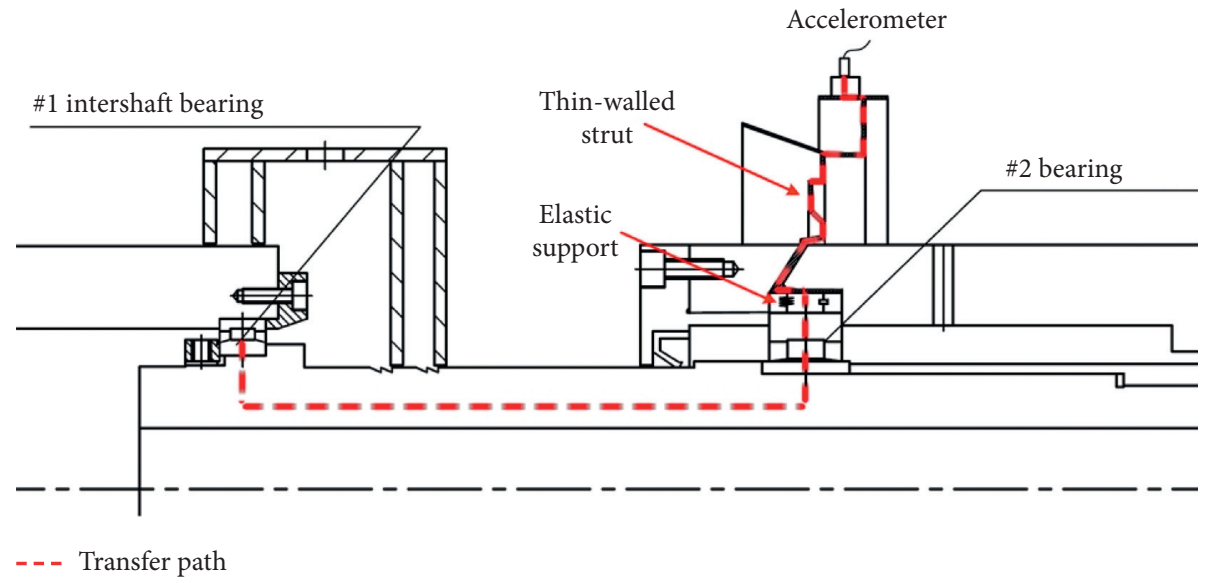

FIgURE 16: Transfer path of vibration signal of \#1 intershaft bearing.

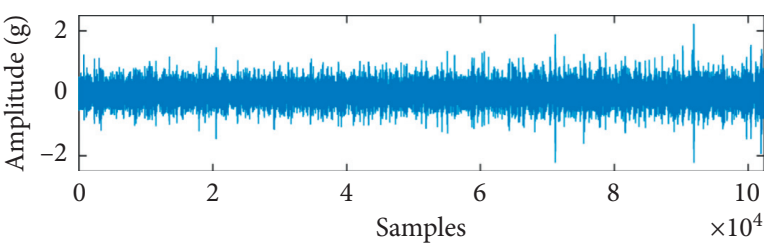

(a)

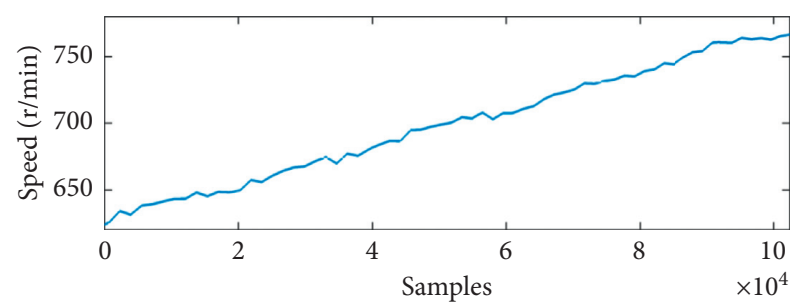

(b)

FIGURE 17: Fault in the outer race: (a) waveform of vibration; (b) speed trend.

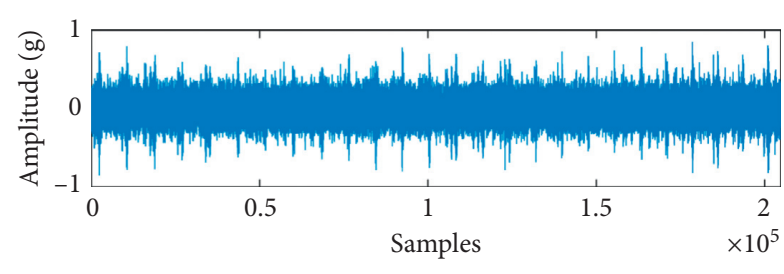

(a)

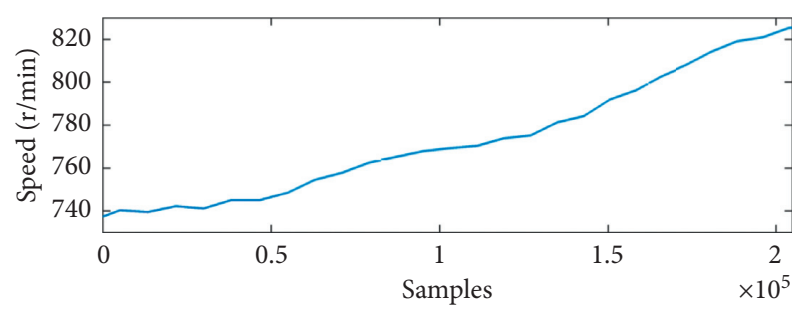

(b)

Figure 18: Fault in the inner race: (a) waveform of vibration; (b) speed trend.

5.2. Fault Characteristic Frequencies of Rolling Bearing. In the experiment, geometrical parameters of the faulty bearing are listed in Table 3, and the fault characteristic orders are shown in Table 4.

\subsection{Compressive Extraction of Fault Feature by MCM-CS}

5.3.1. Outer Race Fault Detection. The proposed method is first utilized to extract the faulty feature of 1 \# intershaft bearings with an outer race fault. As presented in Figure 17, the original vibration signal has intense timedomain nonstationarity and the number of vibration signals for fault extraction is a bit larger, which increases the difficulty of fault feature extraction based on CS. Firstly, the original vibration signal is order tracked to obtain the stationary signal in the angle domain. Compared with the original signal, the stationarity of the angular signal in Figure 19 is greatly enhanced. Although there are still more than 23000, the sample is compressed by $77.6 \%$ while retaining the faulty feature of the bearing, which eases the pressure of further compression by CS.

PSO is utilized to analyze the outer race fault signal in Figure 19. The maximum value of local energy ratio changes with the evolution algebra as shown in Figure 20. The main parameters' setting and optimization results are presented in Table 5 . The optimal combination of $[\alpha, K]$ obtained by optimization is $[65,3]$.

The angular signal is decomposed by VMD, where the penalty factor and decomposition numbers are artificially set to be 65 and 3, respectively. Table 6 shows three modes obtained by VMD decomposition, the fitness value of each mode. It indicates that mode 3 is the best mode corresponding to the maximum energy ratio. 
TABLE 3: Geometrical parameters of 1\# intershaft bearing.

\begin{tabular}{lccc}
\hline $\begin{array}{l}\text { Pitch diameter } \\
\mathrm{D}(\mathrm{mm})\end{array}$ & $\begin{array}{c}\text { Ball diameter } \\
\mathrm{d}(\mathrm{mm})\end{array}$ & $\begin{array}{c}\text { Contact } \\
\text { angle } \alpha\left({ }^{\circ}\right)\end{array}$ & $\begin{array}{c}\text { Number of } \\
\text { rolling elements } z\end{array}$ \\
\hline 125 & 8 & 0 & 34 \\
\hline
\end{tabular}

TABLE 4: Fault characteristic orders of 1\# intershaft bearing.

\begin{tabular}{lcccc}
\hline Component & Outer race & Inner race & Rolling elements & Cage \\
\hline Order & 15.9 & 18.1 & 7.8 & 0.5 \\
\hline
\end{tabular}

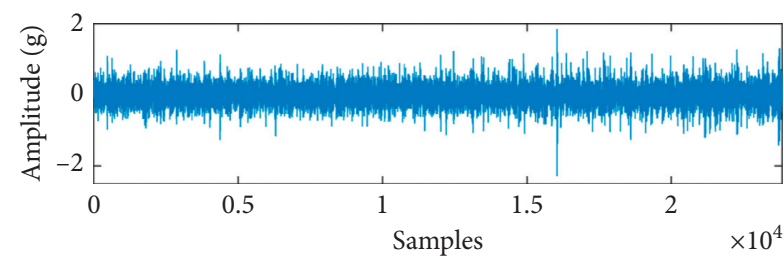

FIgURE 19: The angular signal of outer race fault.

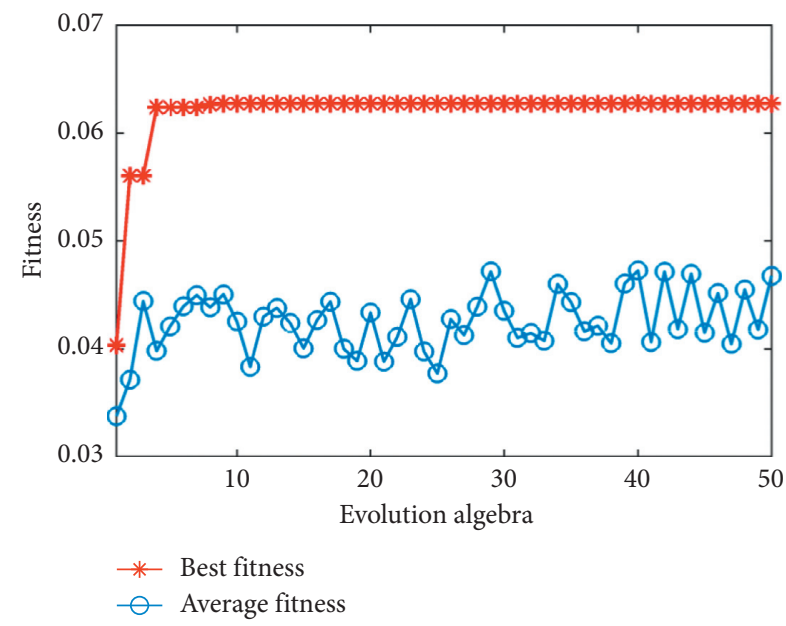

Figure 20: The trend of local energy ratio with evolution algebra.

Therefore, mode 3, as depicted in Figure 21, will replace the angular signal shown in Figure 19 for compression detection of fault feature.

Modes obtained by VMD are continued to be used to construct the MCM, in the detection of the outer-race faulty bearing based on CS. The study selects the number of measurements to be 1500 . Next, the amount of the analyzed signal is compressed to 1500 sampling points assisted by MCM and GRM, respectively.

Based on the constructed MCM, the fault feature is detected by orthogonal matching pursuit with the help of the Fourier dictionary and setting sparsity $K=4$. As provided in Table 4, the theoretical fault feature order of the outer race fault is 15.9. The detected result is illustrated in Figure 22(a), where 239.7 and 255.6 are the sparse coefficients under the Fourier dictionary, and the difference between them is 15.9.

Furthermore, the Fourier dictionary is used to transform the detected result in Figure 22(a) into the recovered signal, and envelope demodulation is carried out to obtain the order spectrum which can directly recognize the fault feature, as presented in Figure 22(b). Thus, since the extracted result is equal to the theoretical one, a conclusion can be drawn that there is a fault in the outer race of $1 \#$ intershaft bearing.

5.3.2. Inner Race Fault Detection. To fully validate the effectiveness of the proposed fault feature extraction strategy, 1 \# intershaft bearing with a faulty inner race was employed. Firstly, the raw vibration signal of inner race fault in Figure 18 is processed by order tracking to obtain the angular vibration signal, the waveform of which is as shown in Figure 23.

PSO is utilized to analyze the inner race fault signal in Figure 24. A fitness curve can be obtained, as shown in Figure 24. The main parameters' setting and optimization results are shown in Table 7 . The optimal combination of $[\alpha$, $K]$ obtained by optimization is $[109,5]$.

Five modes are obtained by VMD decomposition with penalty factor 109 and the decomposition numbers 5, which are prepared for denoising and constructing the MCM. Among the modes, mode 2 is the best component corresponding to the maximum energy ratio, so mode 2 is the main mode (Table 8 . The waveform of mode 2 is shown in Figure 25.

Next, the MCM and GRM are used as a measurement matrix to acquire low-dimensional signals with the measurement numbers set to 1500 , respectively. When the sparsity is determined as 4 , the coefficients corresponding to the faulty feature can be detected from the compressed signal by orthogonal matching pursuit with the sensing matrix constructed by MCM and Fourier dictionary. Next, the detected result, as shown in Figure 26(a), can be recovered by the Fourier dictionary. Finally, the recovered signal can be demodulated by envelope analysis to obtain the envelope order spectrum, as presented in Figure 26(b). The previous contains the modulated frequency order 162.8 and sideband 18.1 which are almost equal to the theoretical one of the inner fault feature. Moreover, the faulty feature order 18.09 and its twice value are extracted directly in the envelope order spectrum. Thus, the judgment that there is an innerrace fault in $1 \#$ intershaft bearing can be concluded.

\subsection{Effect Comparison of Different Methods}

5.4.1. Compressive Extraction of Fault Feature by GRM-CS. To further highlight the high sensing efficiency of MCM and the advantages of MCM-CS in signal compression and feature extraction, the GRM-based CS (GRM-CS) is selected as a comparison. The following steps can be used:

(1) Signal preprocessing and sparsity enhancement: the same is as in Sections 3.1 and 3.2.

(2) Compressive sensing: the GRM is utilized to reduce the dimensions of the denoised signal, and the Fourier dictionary and GRM make up the sensing matrix; finally, the sparse vector is searched by orthogonal matching pursuit. 
TABLe 5: Parameters setting and optimization results of PSO.

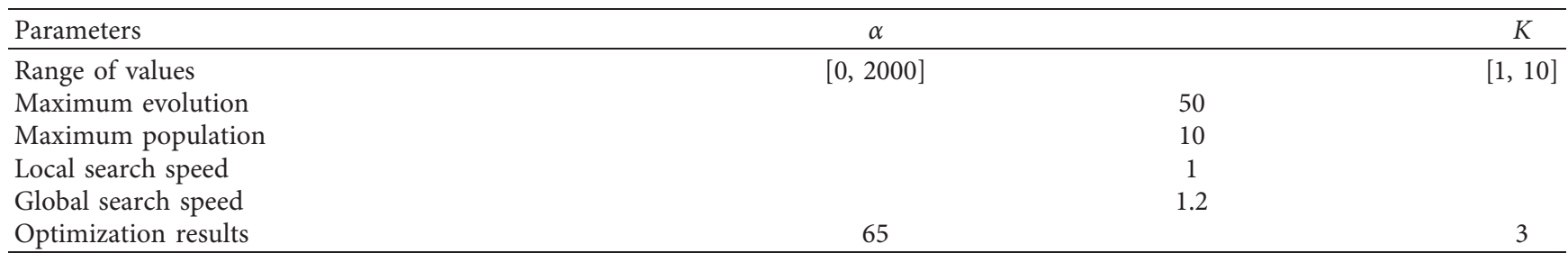

TAвLE 6: Fitness values obtained by analyzing the outer race fault signal with VMD.

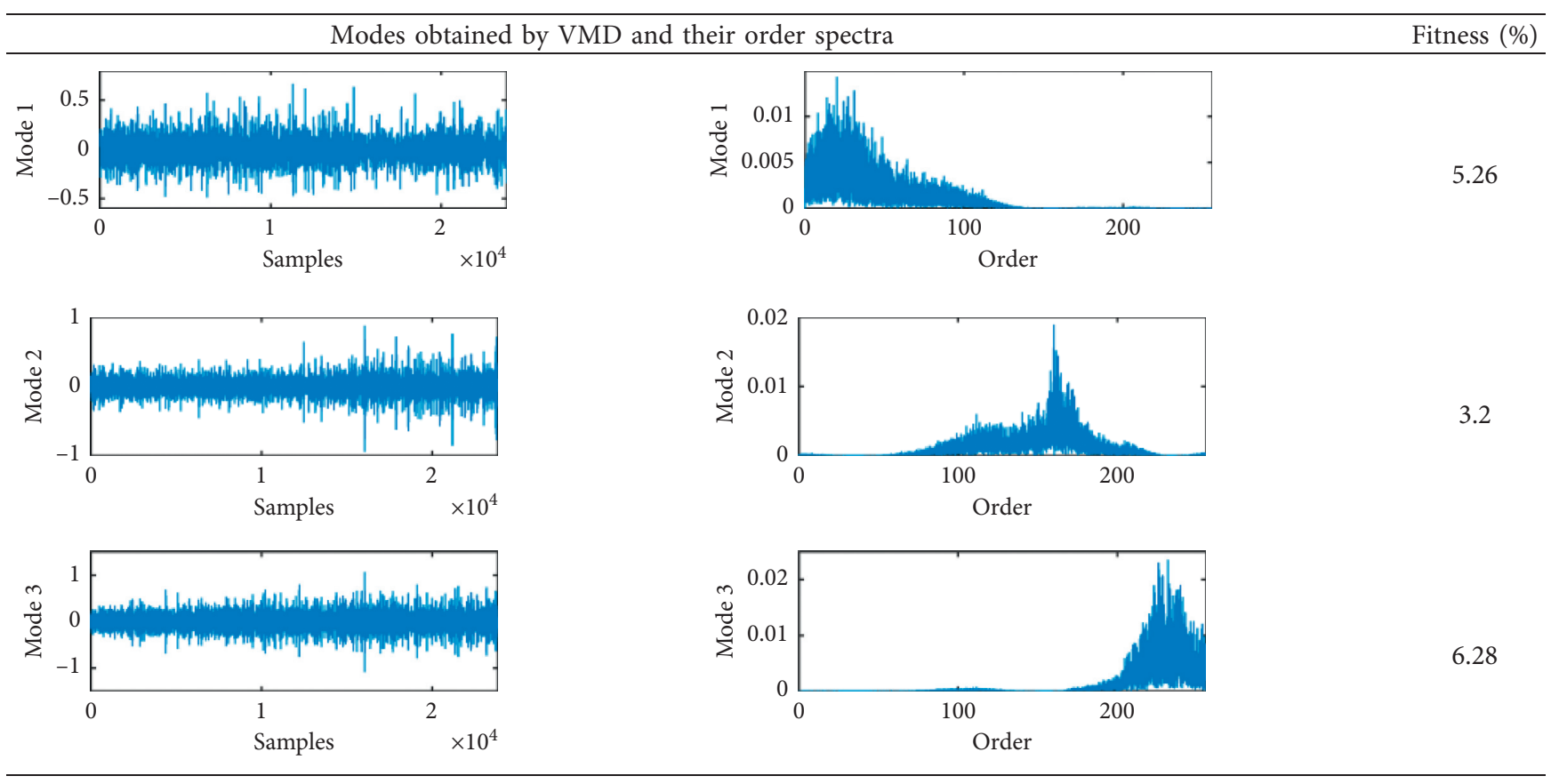

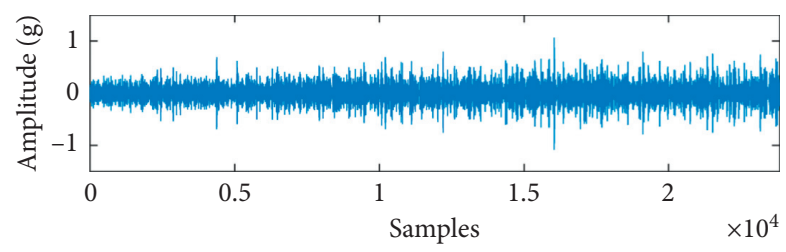

Figure 21: The denoised signal of outer race fault.

(3) Fault feature extraction: the signal recovery can be achieved using the Fourier dictionary and the sparse vector. Because of amplitude modulation of the recovered signal, the envelope demodulation is applied to achieve the envelope order spectrum.

Thus, the envelope order spectrum is obtained, as shown in Figures 27 and 28.

Figure 27 shows the envelope order spectrum of the fault signal of the intershaft bearing's outer race, in which the dominant order is 58.18. An order of 15.9, corresponding to a fault in the outer race, can be seen but it is not the dominant component.

Figure 29 shows the envelope order spectrum of the fault signal of the intershaft bearing's inner race. However, the order component 18.09 corresponding to the inner-race fault of $1 \#$ intershaft bearing cannot appear in Figure 29, which means the fault feature cannot be extracted through the GRM-based CS.

The analysis of experimental data proves that the proposed MCM-based CS method has higher sensing efficiency than GRM-based CS again. Under the same measurement number, the former can successfully extract the weak fault feature of rolling bearings.

5.4.2. Fault Feature Extraction by NEA Based on SK. To assess the value of the proposed method properly and show that its novelty can be compensated by performance, following the steps in Section 4.3.3, we use NEA based on SK to 


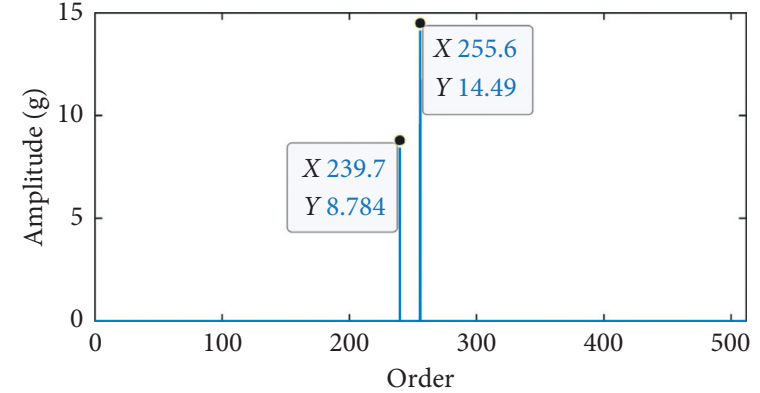

(a)

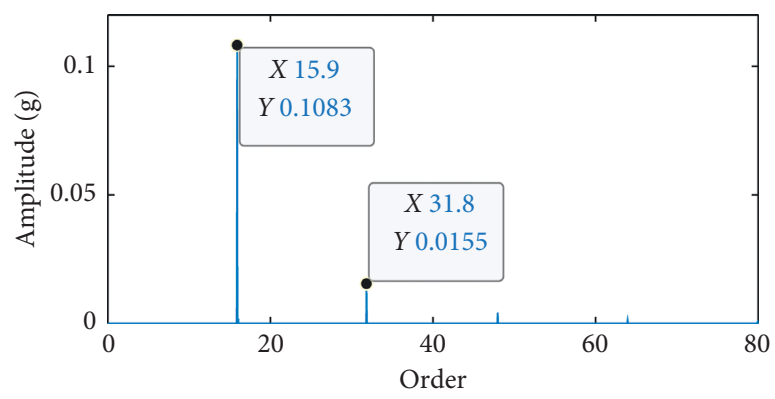

(b)

FIGURE 22: The extracted result of outer race fault using CS-based on MCM: (a) the detected sparse coefficients; (b) the visualized envelope order spectrum.

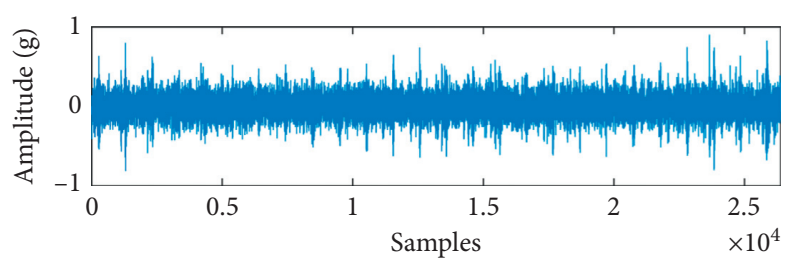

FIGURE 23: The angular signal of inner race fault.

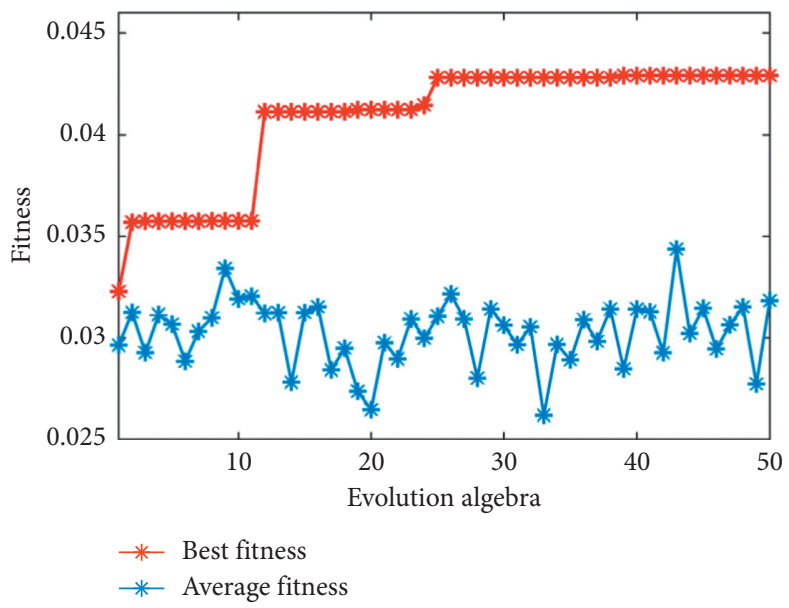

Figure 24: The trend of local energy ratio with evolution algebra.

TABLe 7: Parameters setting and optimization results of PSO.

\begin{tabular}{lcc}
\hline Parameters & $\alpha$ & $K$ \\
\hline Range of values & {$[0,2000]$} & 50 \\
Maximum evolution & 10 \\
Maximum population & 1 \\
Local search speed & 1.2 \\
Global search speed & 109 \\
Optimization results & \\
\hline
\end{tabular}

analyze experimental signals for comparison. However, the following analysis shows that it is hard to achieve significant effects with the SK-based NEA.

Figure 28 shows the analysis results for the fault signal of the 1 \# intershaft bearing's outer race using NEA based on SK. Figure 28(a) is the kurtogram, where the center frequency of the optimum band-pass filter is $2000 \mathrm{~Hz}$ and the bandwidth is $800 \mathrm{~Hz}$. That is, the optimum demodulation band is $[1600,2400] \mathrm{Hz}$. Figure 28(b) shows the envelope order spectrum. In this spectrum, an order of 2.7 has the highest amplitude. The outer race fault feature order 15.9 is fully buried in noise. 
TABLE 8: Fitness values obtained by analyzing inner race fault signal with VMD.

Modes obtained by VMD and their order spectra
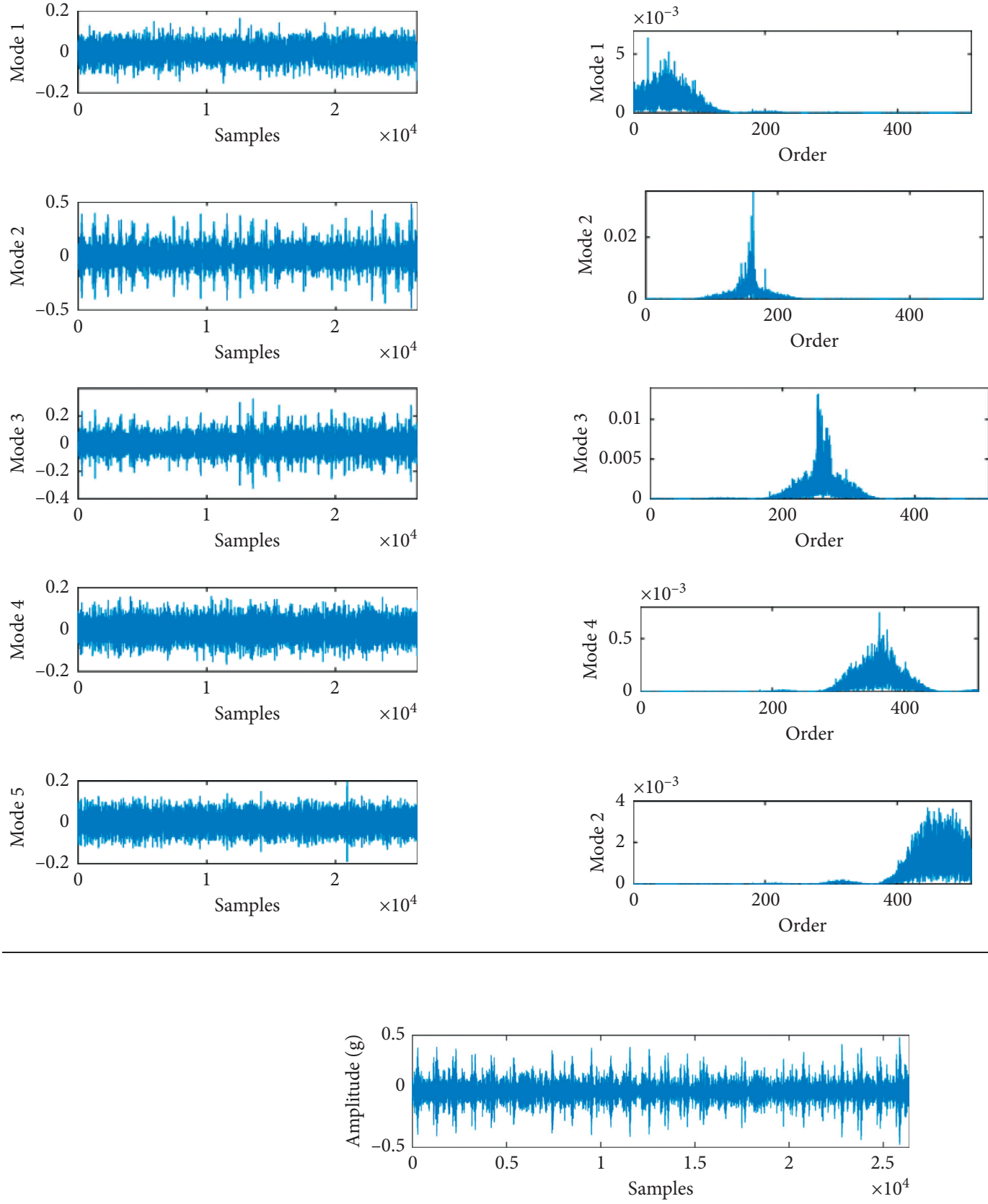

Figure 25: The denoised signal of inner race fault.

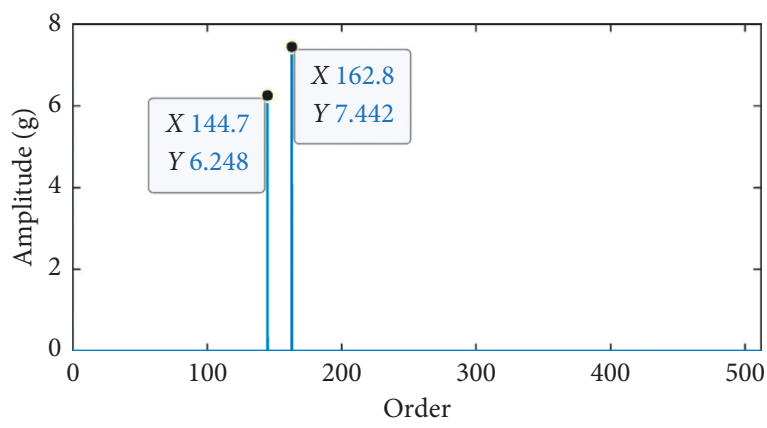

(a)

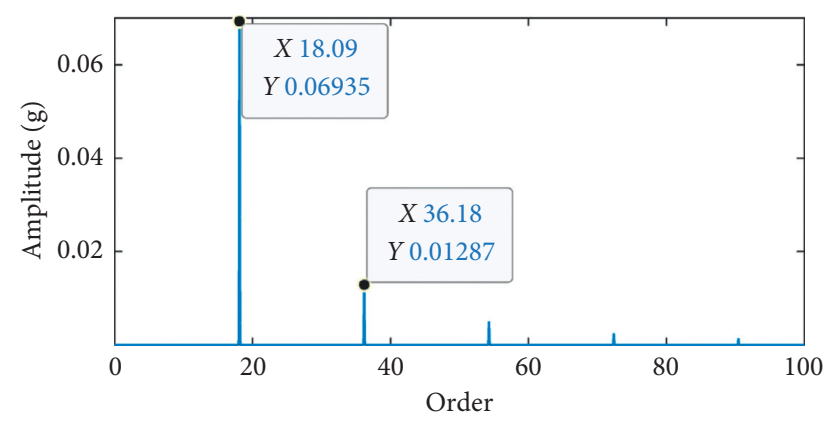

(b)

Figure 26: The extracted result of inner race fault using CS-based on MCM: (a) the detected sparse coefficients and (b) the visualized envelope order spectrum. 


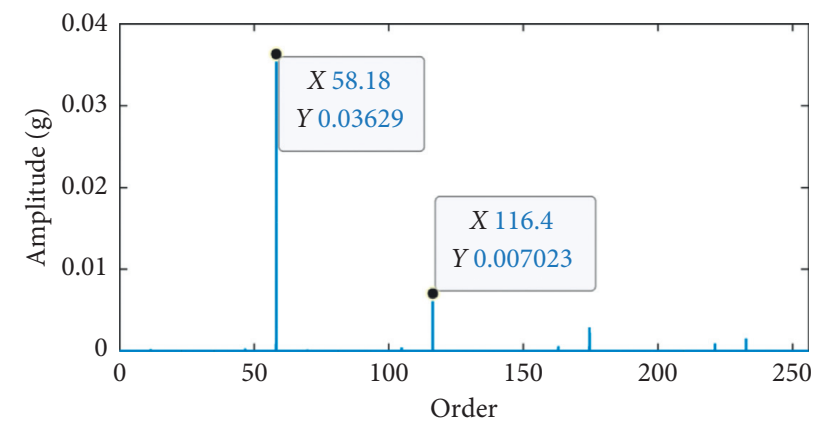

FIGURE 27: The envelope order spectrum of outer race fault using GRM-CS.

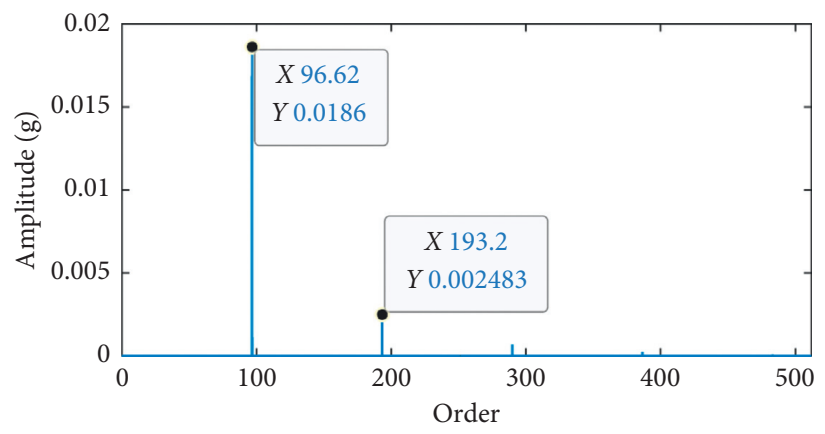

FIGURE 28: The analysis results for fault data of outer race by using NEA based on SK: (a) kurtogram; (b) envelope order spectrum.

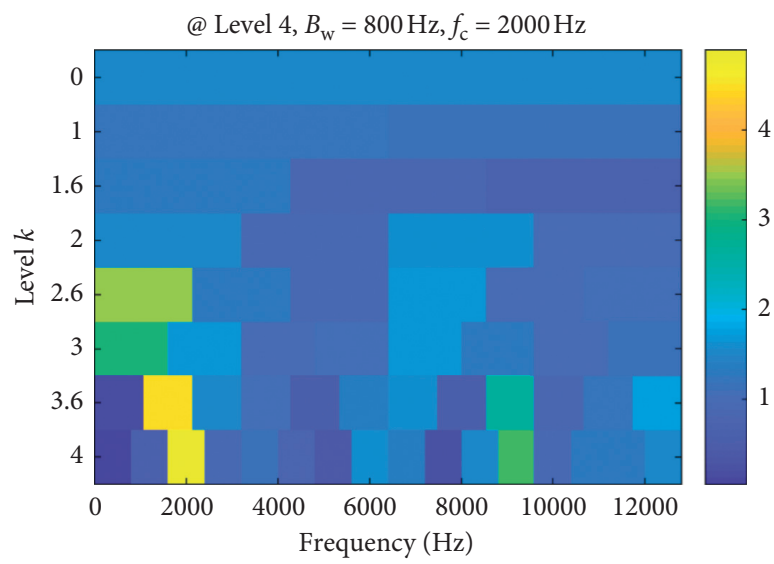

(a)

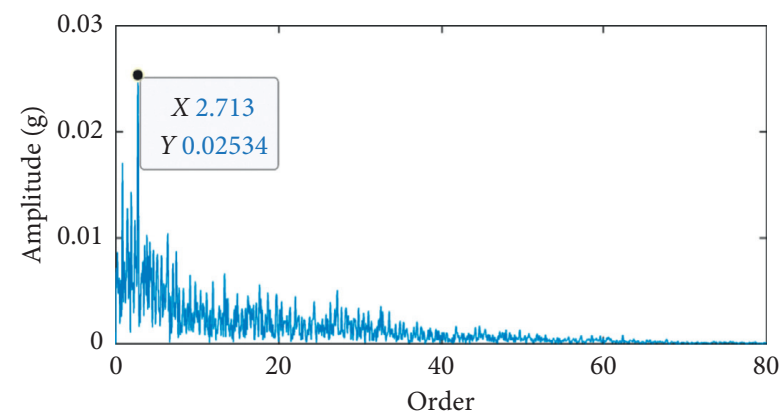

(b)

FIgURE 29: The envelope order spectrum of inner race fault using GRM-CS.

Similarly, Figure 30 shows the analysis results for the fault signal of the inner race. Figure 30 (a) shows that the optimal demodulation frequency band is $[0,25600] \mathrm{Hz}$. Figure $30(\mathrm{~b})$ is the envelope order spectrum; an order of 1 and its harmonic are the dominant components, in which three times harmonic is particularly prominent. An order of 18.1, corresponding to the fault of the bearing inner race, is not effectively extracted.
It can be seen that the order of prominence in Figures 28 and 30 is the result of other impacts in the rotor system. Owing to its existence, NEA based on SK fails to extract the features generated by bearing fault impact. Combined with the analysis of Section 4.3.3, Section 5.3, and Section 5.4.2, it is indicated that SK-based NEA fails to extract the weak fault features of bearings in the presence of random noise and impact noise, which 


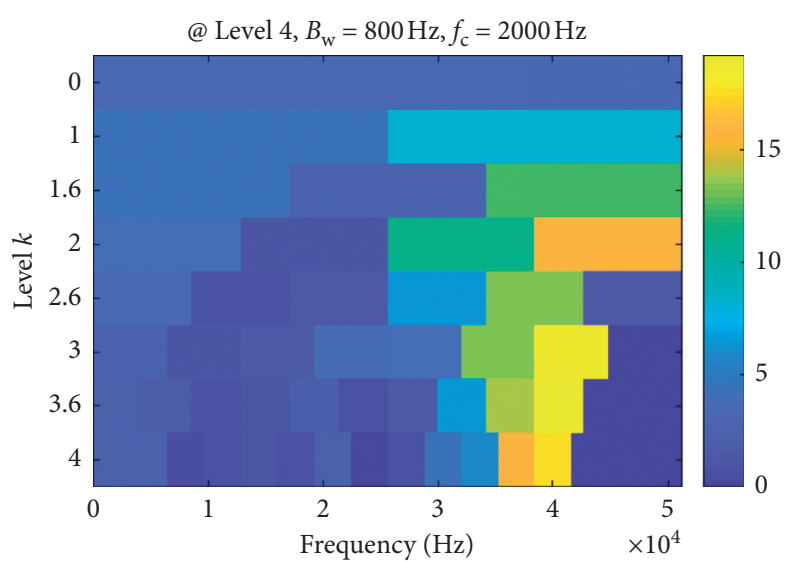

(a)

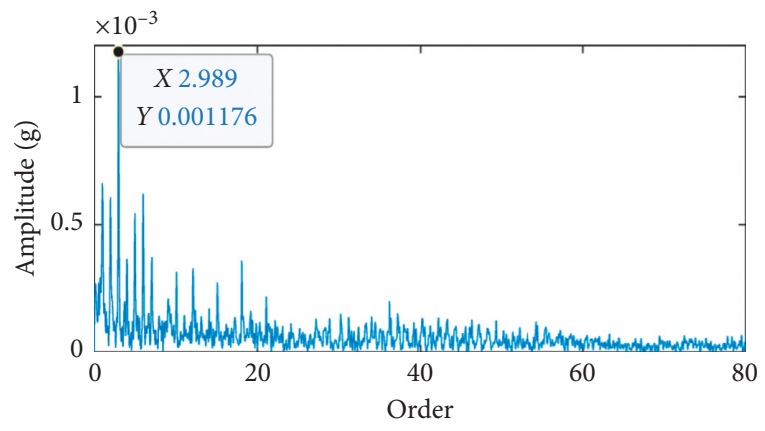

(b)

FIGURE 30: The analysis results for fault data of inner race by using NEA based on SK: (a) kurtogram; (b) envelope order spectrum.

exactly highlights the unique advantages of MCM-CS in face of such difficulties.

\section{Conclusions}

In this paper, a VMD-based CS method is proposed to extract the weak fault feature of rolling element bearings under variable speeds to overcome the inadequate sensing efficiency. First of all, order tracking is employed to eliminate the nonstationarity of the signal caused by the speed change. Secondly, through VMD, the angular vibration signal from the previous step can be decomposed into a series of modes, before which Particle Swarm Optimization algorithm is involved in the optimization of VMD parameters to retain bearing fault signals as much as possible. The maximum energy ratio mode is a great substitute for the next compression detection, because of the enhanced sparsity. In the application of CS, the mode matrix is used to construct MCM as the measurement matrix to compress the denoised signal. Based on the compressed signal, the orthogonal matching pursuit is employed to detect the sparse coefficients corresponding to fault feature where the Fourier dictionary is selected to construct sensing matrix and the sparsity is determined as 4 by signal feature. Finally, for feature visualization, the recovered signal is obtained from the detected result using the Fourier dictionary, and the demodulation is employed to extract the fault feature which characterizes the performance of rolling element bearings. The simulated signals and experimental data are utilized to validate that the sensing efficiency of the proposed method is significantly promoted. Despite the strong background noise, the proposed scheme can successfully extract the weak fault feature of rolling element bearings operating at variable speed.

\section{Data Availability}

The MAT data used to support the findings of this study are available from the corresponding author upon request.

\section{Conflicts of Interest}

The authors declare no conflicts of interest.

\section{Acknowledgments}

This work was supported by the NSFC-Liaoning Joint fund under Grant no. U1708257; Postdoctoral Innovation Talent Support Program (Grant no. BX20180031); and Fundamental Research Fund for the Central Universities (Grant no. JD1913).

\section{References}

[1] R. B. Randall and J. Antoni, "Rolling element bearing diagnostics-a tutorial," Mechanical Systems and Signal Processing, vol. 25, no. 2, pp. 485-520, 2011.

[2] Z. N. Jiang, M. H. Hu, K. Feng et al., "Weak fault feature extraction scheme for intershaft bearings based on linear prediction and order tracking in the rotation speed difference domain," Applied Sciences, vol. 7, no. 9, pp. 1-26, 2017.

[3] R. B. Randall, Vibration-based Condition Monitoring, A John Wiley and Sons, Ltd, Publication, Hoboken, NJ, USA, 2011.

[4] S. C. Dai, Y. Guo, and X. Wu, "Gear-box rolling bearings' fault feature extraction based on cepstrum editing and time domain synchronous average," Journal of Vibration and Shock, vol. 34, no. 21, pp. 205-209, 2015.

[5] H. Li and D.-y. Xiao, "Fault diagnosis based on power spectral density basis transform," Journal of Vibration and Control, vol. 21, no. 12, pp. 2416-2433, 2015.

[6] P. D. McFadden and J. D. Smith, "Vibration monitoring of rolling element bearings by the high-frequency resonance technique - a review," Tribology International, vol. 17, no. 1, pp. 3-10, 1984.

[7] R. B. Randall, "A history of cepstrum analysis and its application to mechanical problems," Mechanical Systems and Signal Processing, vol. 97, pp. 3-19, 2017.

[8] C. Mishra, A. K. Samantaray, and G. Chakraborty, "Rolling element bearing defect diagnosis under variable speed operation through angle synchronous averaging of wavelet denoised estimate," Mechanical Systems and Signal Processing, vol. 72-73, pp. 206-222, 2016. 
[9] N. E. Huang, Z. Shen, S. R. Long et al., "The empirical mode decomposition and the Hilbert spectrum for nonlinear and non-stationary time series analysis," Proceedings of the Royal Society of London. Series A: Mathematical, Physical and Engineering Sciences, vol. 454, no. 1971, pp. 903-995, 1998.

[10] G. Georgoulas, T. Loutas, and C. D. Stylios, "Bearing fault detection based on hybrid ensemble detector and empirical mode decomposition," Mechanical Systems and Signal Processing, vol. 41, no. 1-2, pp. 510-525, 2013.

[11] A. H. Dooley, "A nonabelian version of the Shannon sampling theorem," SIAM Journal on Mathematical Analysis, vol. 20, no. 3, pp. 624-633, 1989.

[12] E. J. Candès, J. Romberg, and J. Tao, Robust Uncertainty Principles: Exact Signal Reconstruction from Highly Incomplete Frequency Information, IEEE Press, Piscataway, NJ, USA, 2006.

[13] D. L. Donoho, "Compressed sensing," IEEE Transactions on Information Theory, vol. 52, no. 4, pp. 1289-1306, 2006.

[14] W. Chan, K. Charan, D. Takhar, K. Kelly, and D. Mittleman, "A single-pixel terahertz imaging system based on compressed sensing," Applied Physics Letters, vol. 93, no. 12, p. S293, 2008.

[15] M. Lustig, D. L. Donoho, J. M. Santos, and J. M. Pauly, "Compressed sensing MRI," IEEE Signal Processing Magazine, vol. 25, no. 2, pp. 72-82, 2008.

[16] X. C. Xie and Y. H. Zhang, "2D radar imaging scheme based on compressive sensing technique," Journal of Electronics \& Information Technolog, vol. 32, no. 5, pp. 1234-1238, 2010.

[17] X. F. Li, X. C. Fan, and L. M. Jia, "Compressed sensing technology applied to fault diagnosis of train rolling bearing," Applied Mechanics and Materials, vol. 226-228, pp. 20562061, 2012.

[18] X. P. Zhang, N. Q. Hu, and Z. Cheng, A Bearing Fault Detection Method Based on Compressed Sensing. Engineering Asset Management-Systems. Professional Practices and Certification, Springer International Publishing, Berlin, Germany, 2015.

[19] X. Chen, Z. Du, J. Li, X. Li, and H. Zhang, "Compressed sensing based on dictionary learning for extracting impulse components," Signal Processing, vol. 96, pp. 94-109, 2014.

[20] G. Tang, Q. Yang, H.-Q. Wang, G.-G. Luo, and J.-W. Ma, "Sparse classification of rotating machinery faults based on compressive sensing strategy," Mechatronics, vol. 31, pp. 6067, 2015.

[21] H. Q. Wang, Y. L. Ke, and L. Y. Song, "A sparsity-promoted decomposition for compressed fault diagnosis of roller bearings," Sensors, vol. 16, no. 9, pp. 1-20, 2016.

[22] H. Y. Guo, T. J. Wang, and Z. Yang, "Adaptive speech compressed sensing in the DCT domain," Chinese Journal of Scientific Instrument, vol. 31, pp. 1262-1268, 2010.

[23] A. Abtahi and M. Modarreshashemi, "Power allocation and measurement matrix design for block CS-based distributed MIMO radars," Aerospace Science and Technology, vol. 53, pp. 128-135, 2015.

[24] S. H. S. Masoumian and B. M. Tazehkand, "On joint compressed sensing based channel estimation and nonuniform PTS PAPR reduction without side information," National Academy Science Letters, vol. 39, no. 6, pp. 445-449, 2016.

[25] E. J. Candès, "Compressive sampling," Marta Sanz Solé, vol. 17, no. 2, pp. 1433-1452, 2006.

[26] K. Dragomiretskiy and D. Zosso, "Variational mode decomposition," IEEE Transactions on Signal Processing, vol. 62, no. 3, pp. 531-544, 2014.
[27] H. Mahgoun, A. Felkaoui, and S. Fedala, "Detection of gear faults in variable rotating speed using variational mode decomposition (VMD)," Mechanics \& Industry, vol. 17, pp. 178-195, 2016.

[28] M. Zhang, Z. Jiang, and K. Feng, "Research on variational mode decomposition in rolling bearings fault diagnosis of the multistage centrifugal pump," Mechanical Systems and Signal Processing, vol. 93, pp. 460-493, 2017.

[29] W. Sintunavarat, "The upper bound estimation for the spectral norm of $\mathrm{r}$-circulant and symmetric $\mathrm{r}$-circulant matrices with the Padovan sequence," Journal of Nonlinear Sciences and Applications, vol. 09, no. 01, pp. 92-101, 2016.

[30] J. F. Guo, J. X. Shi, C. L. Lei, and X. Wei, "Data compression method for collecting rolling bearing vibration signals," Journal of Vibration and Shock, vol. 34, no. 23, pp. 8-13, 2015.

[31] R. B. Randall and J. Antoni, "The relation between spectral correlation and envelope analysis for cyclostationary machine signals, application to ball bearing diagnostics," Mechanical Systems and Signal Processing, vol. 15, no. 5, pp. 945-962, 2011.

[32] F. Cong, J. Chen, G. Dong, and M. Pecht, "Vibration model of rolling element bearings in a rotor-bearing system for fault diagnosis," Journal of Sound and Vibration, vol. 332, no. 8, pp. 2081-2097, 2013.

[33] X. Tian, J. Xi Gu, I. Rehab, G. M. Abdalla, F. Gu, and A. D. Ball, "A robust detector for rolling element bearing condition monitoring based on the modulation signal bispectrum and its performance evaluation against the Kurtogram," Mechanical Systems and Signal Processing, vol. 100, pp. 167-187, 2018.

[34] J. Zhang, J. Yang, H. Liu, and D. Zhou, "Improved SNR to detect the unknown characteristic frequency by SR," IET Science, Measurement \& Technology, vol. 12, no. 6, pp. 795801, 2018.

[35] Y. Wang, J. Xiang, R. Markert, and M. Liang, "Spectral kurtosis for fault detection, diagnosis and prognostics of rotating machines: a review with applications," Mechanical Systems and Signal Processing, vol. 66-67, pp. 679-698, 2016.

[36] 2017 Siemens, LMS SCADAS [EB/OL], https://www.plm. automation.siemens.com/zh/products/lms/testing//scadas/ lab.shtml. 